\title{
Radon Transform: Dual Pairs and Irreducible Representations
}

\author{
Giovanni S. Alberti and Francesca Bartolucci and Filippo De Mari and Ernesto \\ De Vito
}

\begin{abstract}
We illustrate the general point of view developed in [SIAM J. Math. Anal., 51(6), 4356-4381] that can be described as a variation of Helgason's theory of dual $G$-homogeneous pairs $(X, \Xi)$ and which allows us to prove intertwining properties and inversion formulae of many existing Radon transforms. Here we analyze in detail one of the important aspects in the theory of dual pairs, namely the injectivity of the map label-to-manifold $\xi \rightarrow \hat{\xi}$ and we prove that it is a necessary condition for the irreducibility of the quasi-regular representation of $G$ on $L^{2}(\Xi)$. We further explain how the theory in [SIAM J. Math. Anal., 51(6), 4356-4381] applies to the classical Radon and X-ray transforms in $\mathbb{R}^{3}$.
\end{abstract}

Key words: Homogeneous spaces, Radon transform, dual pairs, square-integrable representations, inversion formula, wavelets, shearlets.

G.S. Alberti

Department of Mathematics \& MaLGa center, University of Genoa, Via Dodecaneso 35, 16146

Genova, Italy, e-mail:alberti@dima.unige.it

F. Bartolucci

Department of Mathematics, ETH Zurich, Raemistrasse 101, 8092 Zurich, Switzerland, e-mail: francesca.bartolucci@sam.math.ethz.ch

F. De Mari

Department of Mathematics \& MaLGa center, University of Genoa, Via Dodecaneso 35, 16146 Genova, Italy, e-mail: demari@dima.unige.it

E. De Vito

Department of Mathematics \& MaLGa center, University of Genoa, Via Dodecaneso 35, 16146 Genova, Italy, e-mail:ernesto.devito@unige.it 


\section{Introduction}

The circle of ideas and problems that may be collectively named "Radon transform theory" was born at least a century ago [20] but still abounds with questions and new perspectives that range from very concrete computation-oriented tasks to geometric or representation theoretic issues. We may describe the heart of the matter by paraphrasing Gelfand [10]:

"Let $X$ be some space and in it let there be given certain manifolds which we shall suppose to be analytic and dependent analytically on parameters $\xi_{1}, \ldots, \xi_{k}$, that is $\left\{\hat{\xi}(\xi)=\hat{\xi}\left(\xi_{1}, \ldots, \xi_{k}\right)\right\}$. With a function $f$ on $X$ we associate its integrals over these manifolds:

$$
\mathcal{R} f(\xi)=\int_{\hat{\xi}} f(x) \mathrm{d} m_{\xi}(x) .
$$

We then ask whether it is possible to determine $f$ knowing the integrals $\mathcal{R} f(\xi)$."

Among the many generalizations and theorems that may be subsumed in this basic, yet profound, mathematical sketch, it is certainly worth mentioning Helgason's contribution, inspired [14] by work of Fritz John's, in turn triggered by Radon's original result [20] dating back to 1917. In particular, Helgason developed the notion of dual pairs and double fibrations, whereby (Lie) groups and homogeneous spaces thereof stand at center stage. His basic observation comes by inspecting John's inversion formula for the integral transform-nowadays the prototypical Radon transform-defined by integration over planes in $\mathbb{R}^{3}$. The inversion takes the form

$$
f(\mathbf{x})=-\frac{1}{8 \pi^{2}} \Delta_{\mathbf{x}}\left(\int_{S^{2}} \mathcal{R} f(\mathbf{n}, \mathbf{n} \cdot \mathbf{x}) \mathrm{d} \mathbf{n}\right),
$$

where $(\mathbf{n}, t) \mapsto \mathcal{R} f(\mathbf{n}, t)$ is the function on $S^{2} \times \mathbb{R}$ given by the integral of $f$ over the plane $\hat{\xi}_{\mathbf{n}, t}=\left\{\mathbf{x} \in \mathbb{R}^{3}: \mathbf{n} \cdot \mathbf{x}=t\right\}, \Delta_{\mathbf{x}}$ is the Laplacian and dn is the Riemannian measure on the sphere $S^{2}$. This formula, observes Helgason [14], "involves two dual integrations, $\mathcal{R} f$ is the integral over the set of points in a plane and then $\mathrm{dn}$, the integral over the set of planes through a point." Furthermore, the domain $X$ on which the functions of interest are defined (here $X=\mathbb{R}^{3}$ ) and the set $\Xi$ of relevant manifolds (here the two-dimensional planes) are homogeneous spaces of the same group $G$, namely the group of isometries of $\mathbb{R}^{3}$, and enjoy a sort of duality, well captured by the differential-geometric notion of incidence that was introduced by Chern [6].

Helgason proceeds on developing this duality in group-theoretic terms, emphasizing a remarkable formal symmetry, according to which the objects of interest come naturally in pairs, one living in $X$ and its twin in $\Xi$. Most notably, each point $\xi \in \Xi$ (the pair $\left(\xi_{1}, \xi_{2}\right)=(\mathbf{n}, t)$ in our basic example) labels one of the actual submanifolds $\hat{\xi}$ of $X$ on which the relevant integrals are to be taken (the plane $\hat{\xi}(\mathbf{n}, t)$ ). Conversely, with each point $x \in X$ it is natural to associate the "sheaf" of planes passing through it. In the example above, this is precisely the set $\check{\mathbf{x}}=\left\{\hat{\xi}(\mathbf{n}, \mathbf{x} \cdot \mathbf{n}): \mathbf{n} \in S^{2}\right\}$ over which the integral of $\mathcal{R} f$ is taken. 
In the abstract setting developed by Helgason, the whole construction enjoys natural properties as long as the mappings $\xi \mapsto \hat{\xi}$ and $x \mapsto \check{x}$ are both injective, requirement that is then built into the definition of dual pair and expressed algebraically. Note that in the above example, the map $(\mathbf{n}, t) \mapsto \hat{\xi}(\mathbf{n}, t)$ is two-to-one and this lack of injectivity is reflected by the fact that $\mathcal{R} f$ is an even function. The central object is of course the Radon transform

$$
\mathcal{R} f(\xi)=\int_{\hat{\xi}} f(x) \mathrm{d} m_{\xi}(x)
$$

for integrable functions on $X$, where $m_{\xi}$ is a suitable measure on $\hat{\xi}$.

Utilizing a variation of this framework, which is recalled in full detail below, we have addressed [1] some issues that are naturally expressed in this language. Our main contribution (see Theorem 1 i) is a general result concerning the "unitarization" of $\mathcal{R}$ from $L^{2}(X, \mathrm{~d} x)$ to $L^{2}(\Xi, \mathrm{d} \xi)$ and the fact that the resulting unitary operator intertwines the quasi regular representations $\pi$ and $\hat{\pi}$ of $G$ on $L^{2}(X, \mathrm{~d} x)$ and $L^{2}(\Xi, \mathrm{d} \xi)$, respectively. This unitarization really means first pre-composing the closure of $\mathcal{R}$ with a suitable pseudo-differential operator and then extending this composition to a unitary map, as is done in the existing and well-known precedecessors of Theorem 1 , such as those in [13] and in [23]. The representations $\pi$ and $\hat{\pi}$ of course play a central role and are assumed to be irreducible, and $\pi$ is assumed to be square integrable (see assumptions A4) and A5) below). The combination of unitary extension and intertwining leads to an interesting inversion formula for the true Radon transform, see Theorem 2

Compared to [1], the present article adopts a slightly different, though fully compatible, formalism in the sense that we take here the point of view that seems most natural in applications. Indeed, the space $X$ where the signals of interest are defined and the set of submanifolds of $X$ where integrals are to be taken are both in the foreground, and the group $G$ of geometric actions that one wants to consider comes next, taylored to the problem at hand. In this regard, it is important to observe that, in principle, there are many different realizations of $X$ as homogeneous space, and the choice of $G$ is tantamount to choosing the particular set of transformations (or symmetries) that one wants to focus on. What is of course essential is that they are plentiful enough. As for the submanifolds, we observe that in most applications one has in mind a prototypical submanifold $\hat{\xi}_{0}$. We thus choose and fix $\hat{\xi}_{0}$, which we refer to as the root submanifold, as the image of the base point $x_{0} \in X$ under the action of some closed subgroup $H$ of $G$. Thus $\hat{\xi}_{0}=H\left[x_{0}\right]$, and the other submanifolds are obtained by exploiting the fact that $X$ is a transitive $G$-space. This entails that $X$ is covered with all the shifted versions of $\hat{\xi}_{0}$ by means of the geometric transformations given by the elements of $G$. Incidentally, in this way one often achieves families of foliations, and in most cases this leads to a natural splitting of the parameters in $\Xi$, those that label the foliation and those that select the leaf in the foliation.

Although largely inspired by the work of Helgason, our approach is different in several ways that are discussed in detail in Section 2 His construction rests not only 
on the strict invariance of the measures on $X, \Xi$ and $\hat{\xi}_{0}$ (versus relative invariance as in our construction) but on the fact that the correspondence $\xi \rightarrow \hat{\xi}$ between "labels" in the transitive $G$-space $\Xi$ and submanifolds of $X$ is assumed to be injective. In the present article we investigate this issue in detail and focus on the subgroup $\widetilde{H}$ of $G$ that fixes $\hat{\xi}_{0}$, in principle larger than $H$. We find (Proposition 1) that the map $\xi \rightarrow \hat{\xi}$ is injective if and only if $\widetilde{H}=H$ and we further show in Theorem 3 that, under reasonable assumptions on $\widetilde{H}$, if this equality fails, then $\hat{\pi}$ cannot be irreducible. This implies that in order for assumption A5) to be fulfilled, one must choose $H$ as large as possible among those subgroups of $G$ that fill out $\hat{\xi}_{0}$ by acting on $x_{0}$. Our theory is then illustrated with the help of two examples, namely the classical Radon transform and the X-ray transform in $\mathbb{R}^{3}$, both analyzed with the group $\operatorname{SIM(3)}$ of rotations, dilations and translations. Again, this is different from Helgason's standard choice, the isometry group $\mathrm{M}(3)$.

The paper is organized as follows. In Section 2 we set up the context and recall the main results of [1]. In Section 3 we present a rather detailed analysis of the relations existing between the objects naturally arising from an arbitrary choice of $H$ and those that come from the maximal choice $\widetilde{H}$. This leads to the main contribution of this work, namely the fact that a gap between $\widetilde{H}$ and $H$ implies that the quasi regular representation $\hat{\pi}$ of $G$ on $L^{2}(\Xi)$ cannot be irreducible. Section 4 illustrates our theory with two classical examples in three-dimensional Euclidean space.

\section{The framework}

In this section we introduce the setting and the main result of [1].

\subsection{Notation}

We briefly introduce the notation. We set $\mathbb{R}^{\times}=\mathbb{R} \backslash\{0\}$ and $\mathbb{R}^{+}=(0,+\infty)$. The Euclidean norm of a vector $v \in \mathbb{R}^{d}$ is denoted by $|v|$ and its scalar product with $w \in \mathbb{R}^{d}$ by $v \cdot w$. For any $p \in[1,+\infty]$ we denote by $L^{p}\left(\mathbb{R}^{d}\right)$ the Banach space of functions $f: \mathbb{R}^{d} \rightarrow \mathbb{C}$ that are $p$-integrable with respect to the Lebesgue measure $\mathrm{d} x$ and, if $p=2$, the corresponding scalar product and norm are $\langle\cdot, \cdot\rangle$ and $\|\cdot\|$, respectively. If $E$ is a Borel subset of $\mathbb{R}^{d},|E|$ denotes its Lebesgue measure. The Fourier transform is denoted by $\mathcal{F}$ both on $L^{2}\left(\mathbb{R}^{d}\right)$ and on $L^{1}\left(\mathbb{R}^{d}\right)$, where it is defined by

$$
\mathcal{F} f(\omega)=\int_{\mathbb{R}^{d}} f(x) \mathrm{e}^{-2 \pi i \omega \cdot x} \mathrm{~d} x, \quad f \in L^{1}\left(\mathbb{R}^{d}\right) .
$$

If $G$ is a locally compact second countable (lcsc) group, we denote by $L^{2}\left(G, \mu_{G}\right)$ the Hilbert space of square-integrable functions with respect to a left Haar measure $\mu_{G}$ on $G$. If $X$ is a lcsc transitive $G$-space with origin $x_{0}$, we denote by $g[x]$ the 
action of $G$ on $X$. A Borel measure $v$ on $X$ is relatively invariant if there exists a positive character $\alpha$ of $G$ such that for any measurable set $E \subseteq X$ and $g \in G$ it holds $v(g[E])=\alpha(g) v(E)$. Furthermore, a Borel section is a measurable map $s: X \rightarrow G$ satisfying $s(x)\left[x_{0}\right]=x$ and $s\left(x_{0}\right)=e$, with $e$ the neutral element of $G$; a Borel section always exists since $G$ is second countable [25, Theorem 5.11]. We denote the (real) general linear group of size $d \times d$ by $\mathrm{GL}(d, \mathbb{R})$.

Given two unitary representations $\pi, \hat{\pi}$ of $G$ acting on two Hilbert spaces $\mathcal{H}$ and $\hat{\mathcal{H}}$, respectively, a densely defined closed operator $T: \mathcal{H} \rightarrow \hat{\mathcal{H}}$ is called semi-invariant with weight $\zeta$ if it satisfies

$$
\hat{\pi}(g) T \pi(g)^{-1}=\zeta(g) T, \quad g \in G,
$$

where $\zeta$ is a character of $G$, see [7].

\subsection{Setting and assumptions}

The Radon transform of a signal $f: X \rightarrow \mathbb{C}$ is defined as the integral of $f$ over a suitable family $\{\hat{\xi}\}$ of subsets of $X$ indexed by a label $\xi \in \Xi$.

In this paper, we assume that the input space $X$ is a lcsc space and the signals are elements of the Hilbert space $L^{2}(X, \mathrm{~d} x)$, where $\mathrm{d} x$ is a given measure on $X$, defined on the Borel $\sigma$-algebra of $X$ and finite on compact subsets.

Following Helgason's approach, the family $\{\hat{\xi}\}$ is defined by first choosing a lcsc group $G$ acting on $X$ by a continuous action

$$
(g, x) \mapsto g[x]
$$

in such a way that $X$ becomes a transitive $G$-space. Then, we fix an origin $x_{0} \in X$, a closed subgroup $H$ of $G$ and we define the root $\hat{\xi}_{0}$ of the family $\{\hat{\xi}\}$ as

$$
\hat{\xi}_{0}=H\left[x_{0}\right],
$$

which is a closed $H$-invariant subset of $X$, by [13, Lemma 1.1]. Denote the set of left cosets by $\Xi=G / H$ and define for each $\xi=g H \in \Xi$ the closed subset of $X$

$$
\hat{\xi}=g\left[\hat{\xi}_{0}\right]=g H\left[x_{0}\right]
$$

which is independent of the choice of the representative $g \in G$ of $\xi \in G / H$.

In order to view the roles played by $X$ and $\Xi$ as somewhat symmetric, we introduce the stability subgroup of $G$ at $x_{0}$

$$
K=\left\{k \in G: k\left[x_{0}\right]=x_{0}\right\},
$$

which is a closed subgroup of $G$ such that $X$ can be identified with $G / K$ by means of the map

$$
G / K \ni g K \mapsto g\left[x_{0}\right] \in X .
$$


Conversely, we regard $\Xi$ as a transitive lcsc space with respect to the continuous action of $G$ given by

$$
g . \xi=\left(g g^{\prime}\right) H \quad \xi=g^{\prime} H \in \Xi .
$$

and we choose, as origin, the point $\xi_{0}=e H$, which makes (3) and (4) consistent with each other (see Lemma1 1 below).

With this setting, we need the following conditions to hold true:

A1) the measure $\mathrm{d} x$ is relatively $G$-invariant with character $\alpha$ and there exists a relatively invariant measure $\mathrm{d} \xi$ on $\Xi$ with character $\beta$;

A2) there exists a relatively $H$-invariant measure $m_{0}$ on $\hat{\xi}_{0}$ with character $\gamma$;

A3) there exist a Borel section $\sigma: \Xi \rightarrow G$ for the action (5) and a character $\iota$ of $G$ such that

$$
\gamma\left(\sigma(\xi)^{-1} g \sigma\left(g^{-1} \cdot \xi\right)\right)=\iota(g), \quad g \in G, \xi \in \Xi
$$

A4) the quasi-regular representation $\pi$ of $G$ acting on $L^{2}(X, \mathrm{~d} x)$ as

$$
\pi(g) f(x)=\alpha(g)^{-1 / 2} f\left(g^{-1}[x]\right),
$$

is irreducible and square-integrable;

A5) the quasi-regular representation $\hat{\pi}$ of $G$ acting on $L^{2}(\Xi, \mathrm{d} \xi)$ as

$$
\hat{\pi}(g) F(\xi)=\beta(g)^{-1 / 2} F\left(g^{-1} \cdot \xi\right)
$$

is irreducible;

A6) there exists a non-trivial $\pi$-invariant subspace $\mathcal{A} \subseteq L^{2}(X, \mathrm{~d} x)$ such that for all $f \in \mathcal{A}$

$$
\begin{aligned}
& f(\sigma(\xi)[\cdot]) \in L^{1}\left(\hat{\xi}_{0}, m_{0}\right) \quad \text { for almost all } \xi \in \Xi, \\
& \mathcal{R} f:=\int_{\hat{\xi}_{0}} f(\sigma(\cdot)[x]) \mathrm{d} m_{0}(x) \in L^{2}(\Xi, \mathrm{d} \xi),
\end{aligned}
$$

and the adjoint of the operator $\mathcal{R}: \mathcal{A} \rightarrow L^{2}(\Xi, \mathrm{d} \xi)$ has non-trivial domain.

We add a few comments. The assumption that the measure $\mathrm{d} x$ is (relatively) invariant ensures that the group $G$ acts also on the signals by means of the unitary representation $\pi$.

It is worth observing that $(7 \mathrm{a})$ is independent of the section $\sigma$. Indeed, if $\sigma^{\prime}$ is another section, by assumption A2) we have

$$
\begin{aligned}
\int_{\hat{\xi}_{0}}\left|f\left(\sigma^{\prime}(\xi)[x]\right)\right| \mathrm{d} m_{0}(x) & =\int_{\hat{\xi}_{0}}\left|f\left(\sigma(\xi) \sigma(\xi)^{-1} \sigma^{\prime}(\xi)[x]\right)\right| \mathrm{d} m_{0}(x) \\
& =\gamma\left(\sigma^{\prime}(\xi)^{-1} \sigma(\xi)\right) \int_{\hat{\xi}_{0}}|f(\sigma(\xi)[x])| \mathrm{d} m_{0}(x),
\end{aligned}
$$

since $\sigma(\xi)^{-1} \sigma^{\prime}(\xi) \in H$. 
By means of the section $\sigma$, the family $\{\hat{\xi}\}$ is given by

$$
\hat{\xi}=\sigma(\xi)\left[\hat{\xi}_{0}\right] \subseteq X,
$$

and the map $x \mapsto \sigma(\xi)[x]$ is a Borel bijection from $\hat{\xi}_{0}$ onto $\hat{\xi}$, so that (7b) reads as

$$
\mathcal{R} f(\xi)=\int_{\hat{\xi}} f(x) \mathrm{d} m_{\xi}(x),
$$

where $m_{\xi}$ is the image measure of $m_{0}$ under the above bijection. Hence for any signal belonging to $\mathcal{A}$, the map $\mathcal{R} f$ is precisely the Radon transform of $f$. Note that $\mathcal{A}$ is a dense subspace of $L^{2}(X, \mathrm{~d} x)$ by irreducibility of $\pi$ and this property also guarantees that the adjoint of $\mathcal{R}$ is uniquely defined.

Given the space of signals $L^{2}(X, \mathrm{~d} x)$, there are possibly many different pairs $(G, H)$ that give rise to the same family $\{\hat{\xi}\}$ of subsets and (essentially) to the same Radon trasform $\mathcal{R}$. In this paper, $G$ is chosen in such a way that $\pi$ is a square-integrable representation, so that there exists a self-adjoint operator

$$
C: \operatorname{dom} C \subseteq L^{2}(X, \mathrm{~d} x) \rightarrow L^{2}(X, \mathrm{~d} x),
$$

semi-invariant with weight $\Delta^{\frac{1}{2}}$, where $\Delta$ is the modular function of $G$. Hence, for all $\psi \in \operatorname{dom} C$ with $\|C \psi\|=1$, the voice transform $\mathcal{V}_{\psi}$

$$
\left(\mathcal{V}_{\psi} f\right)(g)=\langle f, \pi(g) \psi\rangle, \quad g \in G,
$$

is an isometry from $L^{2}(X, \mathrm{~d} x)$ into $L^{2}\left(G, \mu_{G}\right)$. In this case the vector $\psi$ is called admissible and we have the weakly-convergent reproducing formula

$$
f=\int_{G}\left(\mathcal{V}_{\psi} f\right)(g) \pi(g) \psi \mathrm{d} \mu_{G}(g),
$$

see, for example, [8, Theorem 2.25]). Eq. (10) is at the basis of our reconstruction formula (14).

We stress that in Helgason's approach, the representation $\pi$ is not directly considered, and hence there is no need to require it to be either irreducible or squareintegrable. This entails a larger freedom in the choice of the group $G$.

We recall that, since $X, \Xi$ and $\hat{\xi}_{0}$ are transitive spaces, there always exist quasiinvariant measures on these three spaces. In Helgason's approach, it is assumed that the measures are invariant, so that they are unique up to a constant. In this paper, we only require that $\mathrm{d} x, \mathrm{~d} \xi$ and $m_{0}$ are relatively invariant. In particular, $m_{0}$ is not uniquely given (up to a constant) and the definition of the Radon transform depends not only on the family $\{\hat{\xi}\}$, but also on the measure $m_{0}$ and the section $\sigma$. Since $m_{0}$ is not invariant, Assumption A3) is needed to ensure the right covariance properties of the Radon transform and in many examples it can be easily satisfied by a suitable choice of the section $\sigma$. 


\subsection{The Unitarization Theorem and Inversion Formula}

The isometric extension problem for the Radon transform was actually addressed and implicitly solved by Helgason in the general context of symmetric spaces, see [12, Corollary 3.11]. However, as a consequence of the intertwining properties of the Radon transform it is possible to provide an alternative proof of the following result, see [1].

Theorem 1 Under the above assumptions,

1. the Radon transform $\mathcal{R}: \mathcal{A} \rightarrow L^{2}(\Xi, \mathrm{d} \xi)$ admits a unique closure $\overline{\mathcal{R}}$;

2. the closure $\overline{\mathcal{R}}$ satisfies

$$
\overline{\mathcal{R}} \pi(g)=\chi(g)^{-1} \hat{\pi}(g) \overline{\mathcal{R}},
$$

for all $g \in G$, where $\chi$ is the character given by

$$
\chi(g)=\alpha(g)^{1 / 2} \beta(g)^{-1 / 2} \gamma\left(g \sigma\left(g^{-1} \cdot \xi_{0}\right)\right)^{-1} ;
$$

3. there exists a unique positive self-adjoint operator

$$
\mathcal{I}: \operatorname{dom}(\mathcal{I}) \supseteq \operatorname{Im} \overline{\mathcal{R}} \rightarrow L^{2}(\Xi, \mathrm{d} \xi),
$$

semi-invariant with weight $\zeta=\chi^{-1}$ with the property that the composite operator $\mathcal{I} \overline{\mathcal{R}}$ extends to a unitary operator $Q: L^{2}(X, \mathrm{~d} x) \rightarrow L^{2}(\Xi, \mathrm{d} \xi)$ intertwining $\pi$ and $\hat{\pi}$, namely

$$
\hat{\pi}(g) Q \pi(g)^{-1}=Q, \quad g \in G .
$$

It follows that the representations $\pi$ and $\hat{\pi}$ are equivalent, so that $\hat{\pi}$ is squareintegrable, too.

Since $Q$ is unitary and satisfies $(39)$ and $\pi$ is square-integrable, it is possible to prove the following inversion formula for the Radon transform, [1].

Theorem 2 Let $\psi \in L^{2}(X, \mathrm{~d} x)$ be an admissible vector for the representation $\pi$ such that $Q \psi \in \operatorname{dom} \mathcal{I}$, and set $\Psi=\mathcal{I} Q \psi$. Then, for any $f \in \operatorname{dom} \overline{\mathcal{R}}$,

$$
f=\int_{G} \chi(g)\langle\overline{\mathcal{R}} f, \hat{\pi}(g) \Psi\rangle \pi(g) \psi \mathrm{d} \mu_{G}(g),
$$

where the integral is weakly convergent, and

$$
\|f\|^{2}=\int_{G} \chi(g)^{2}|\langle\overline{\mathcal{R}} f, \hat{\pi}(g) \Psi\rangle|^{2} \mathrm{~d} \mu(g) .
$$

If, in addition, $\psi \in \operatorname{dom} \overline{\mathcal{R}}$, then $\Psi=\mathcal{I}^{2} \overline{\mathcal{R}} \psi$.

Note that the datum $\overline{\mathcal{R}} f$ is analyzed by the family $\{\hat{\pi}(g) \Psi\}_{g \in G}$ and the signal $f$ is reconstructed by a different family, namely $\{\pi(g) \psi\}_{g \in G}$. The idea to exploit the theory of the continuous wavelet transform to derive inversion formulae for the Radon transform is not new, we refer to [4, 15, 16, 19, 22, 26, 27]-to name a few. 


\section{Dual pairs and irreducibility}

In this section, we show the relation between our setting and the notion of dual pairs introduced by Helgason [13] and the connection with the assumption on the irreducibility. If we identify $X$ and $\Xi$ with the corresponding homogenous spaces $G / K$ and $G / H$, so that $\xi=g_{1} H$ for some $g_{1} \in G$, then a point $x=g_{2} K$ belongs to $\hat{\xi}$ if and only if $g_{2} K \cap g_{1} H \neq \emptyset$, which is the notion of incidence introduced by Chern in [6] and adopted by Helgason.

Interchanging the roles of $X$ and $\Xi$, we can define

$$
\check{x}_{0}=K . \xi_{0} \subseteq \Xi, \quad \check{x}=s(x) \cdot \check{x}_{0} \subseteq \Xi,
$$

where $s: X \rightarrow G$ is any section for the action (2). The notion of incidence makes clear the following duality relation

$$
x \in \hat{\xi} \quad \Longleftrightarrow \quad \xi \in \check{x} .
$$

Furthermore, if $\check{x}_{0}$ admits a relatively invariant measure $\hat{m}_{0}$, we can define the back-projection of a function $\hat{f}: \Xi \rightarrow \mathbb{C}$ as

$$
\mathcal{R}^{\#} \hat{f}(x)=\int_{\check{x}_{0}} \hat{f}(s(x) \cdot \xi) \mathrm{d} \hat{m}_{0}(\xi)=: \int_{\check{x}} \hat{f}(\xi) \mathrm{d} \hat{m}_{x}(\xi),
$$

provided that the integral converges, where $\hat{m}_{x}$ is the image measure of $m_{0}$ under the bijection $\xi \mapsto s(x)$. $\xi$ from $\check{x}_{0}$ onto $\check{x}$.

The pair $(X, \Xi)$ is called a dual pair by Helgason if both the map $\xi \mapsto \hat{\xi}$ and the map $x \mapsto \check{x}$ are injective. Below we provide an alternative characterization of injectivity. We need some preliminary facts.

Lemma 1 For all $g \in G$ and $\xi \in \Xi$

$$
g[\hat{\xi}]=\widehat{g \cdot \xi}
$$

Proof For $g \in G$ and $\xi=g^{\prime} H \in \Xi$, by equations (4) and (5) it holds that

$$
g[\hat{\xi}]=g g^{\prime}\left[\hat{\xi}_{0}\right]=\widehat{g \cdot \xi} .
$$

This concludes the proof.

Lemma 2 The set

$$
\widetilde{H}=\left\{g \in G \mid g\left[\hat{\xi}_{0}\right]=\hat{\xi}_{0}\right\}
$$

is a closed subgroup of $G$ and $\widetilde{H} \supseteq H$.

Proof Clearly, $\widetilde{H}$ is a subgroup of $G$ and $\widetilde{H} \supseteq H$. We prove that it is closed. Let $\left(g_{n}\right)_{n}$ be a sequence of $\widetilde{H}$ converging to $g$ and $x \in \hat{\xi}_{0}$, then $\left(g_{n}[x]\right)_{n}$ is a sequence of $\hat{\xi}_{0}$ converging to $g[x] \in \hat{\xi}_{0}$ since the action is continuous and $\hat{\xi}_{0}$ is closed. Then, 
$g\left[\hat{\xi}_{0}\right] \subseteq \hat{\xi}_{0}$. The same argument applied to the sequence $\left(g_{n}^{-1}\right)_{n}$ in $\widetilde{H}$, which converges to $g^{-1}$, yields $g^{-1}\left[\hat{\xi}_{0}\right] \subseteq \hat{\xi}_{0}$, namely, $\hat{\xi}_{0} \subseteq g\left[\hat{\xi}_{0}\right]$.

The next proposition provides an alternative characterization of the injectivity in terms of $\widetilde{H}$. More precisely, the map $\xi \mapsto \hat{\xi}$ is injective if and only if $H$ is chosen as the maximal subgroups fixing $\hat{\xi}_{0}$. The reader is referred to Section 4 below for two examples of this aspect.

Proposition 1 The map $\xi \mapsto \hat{\xi}$ is injective if and only if $\widetilde{H}=H$.

Proof Given $\xi, \xi^{\prime} \in \Xi$, the condition $\hat{\xi}=\hat{\xi}^{\prime}$ is equivalent to $\sigma\left(\xi^{\prime}\right)^{-1} \sigma(\xi)\left[\hat{\xi}_{0}\right]=\hat{\xi}_{0}$, i.e., $\sigma\left(\xi^{\prime}\right)^{-1} \sigma(\xi) \in \widetilde{H}$.

On the other hand, since $\xi=\sigma(\xi) \cdot \xi_{0}$ and $\xi^{\prime}=\sigma\left(\xi^{\prime}\right) \cdot \xi_{0}$, the condition $\xi=\xi^{\prime}$ is equivalent to $\sigma(\xi) . \xi_{0}=\sigma\left(\xi^{\prime}\right) \cdot \xi_{0}$, i.e., $\sigma\left(\xi^{\prime}\right)^{-1} \sigma(\xi) \in H$.

If $H=\widetilde{H}$, it follows that $\hat{\xi}=\hat{\xi}^{\prime}$ if and only if $\xi=\xi^{\prime}$. If $H \neq \widetilde{H}$, then since $H$ is always contained in $\widetilde{H}$, there exists $g \in \widetilde{H} \backslash H$. Then, by Lemma 1 ,

$$
\hat{\xi}_{0}=g\left[\hat{\xi}_{0}\right]=\widehat{g \cdot \xi_{0}} .
$$

However, $g . \xi_{0} \neq \xi_{0}$ because $g \notin H$.

Since $\widetilde{H}$ is closed, we can consider the transitive space $\widetilde{\Xi}=G / \widetilde{H}$ and, since $H$ is a closed subgroup of $\widetilde{H}$, the map

$$
j: \Xi \rightarrow \widetilde{\Xi}, \quad j(g H)=g \widetilde{H},
$$

is a continuous surjection intertwining the actions of $G$ on $\Xi$ and $\widetilde{\Xi}$, i.e.

$$
j(g . \xi)=g . j(\xi), \quad g \in G, \xi \in \Xi,
$$

where the action of $\widetilde{\Xi}$ is still denoted by $g \cdot \widetilde{\xi}$. Furthermore, for all $\widetilde{\xi}=g \widetilde{H} \in \widetilde{\Xi}$, we define

$$
\widehat{\widetilde{\xi}}=g \widetilde{H}\left[x_{0}\right] .
$$

Corollary 1 For all $\xi \in \Xi$

$$
\widehat{j(\xi)}=\hat{\xi}
$$

and the map $\widetilde{\xi} \mapsto \hat{\xi}$ is injective.

Proof Fix $\xi=g H \in \Xi$, then

$$
\widehat{j(\xi)}=g \widetilde{H}\left[x_{0}\right]=g(\widetilde{H} H)\left[x_{0}\right]=g \widetilde{H}\left[\hat{\xi}_{0}\right]=g\left[\hat{\xi}_{0}\right]=\hat{\xi},
$$

where the second equality holds true since $\widetilde{H} H=\widetilde{H}$ whereas the fourth equality is due to the definition of $\widetilde{H}$. If $\widetilde{\xi_{0}}=j\left(\xi_{0}\right)=\widetilde{H}$ is the origin of $\widetilde{\Xi}$, from (16), it follows that $\underset{\widetilde{\xi_{0}}}{\widetilde{\xi_{0}}} \hat{\xi}_{0}$, so that $\widetilde{\widetilde{H}}=\widetilde{H}$ and the injectivity follows from Prop. 11with $H$ replaced by $\widetilde{H}$. 


\subsection{Irreducibility}

In this section we show that if $H$ is a proper subgroup of $\widetilde{H}$, then $\hat{\pi}$ is not irreducible. To prove the claim we need that $\widetilde{H}$ satisfies the same assumptions made on $H$ and that there is the appropriate compatibility between the two subgroups.

As in A1), we first suppose that $G / \widetilde{H}$ has a $G$-relatively invariant measure $\mathrm{d} \widetilde{\xi}$ with the same character $\beta$ of $\mathrm{d} \xi$. Since $\beta$ satisfies

$$
\beta(h)=\frac{\Delta_{\widetilde{H}}(h)}{\Delta_{G}(h)} \quad h \in \widetilde{H}, \quad \beta(h)=\frac{\Delta_{H}(h)}{\Delta_{G}(h)} \quad h \in H,
$$

then

$$
\Delta_{H}(h)=\Delta_{\widetilde{H}}(h), \quad h \in H .
$$

Eq. (18) implies that there exists an invariant measure $\omega$ on $\widetilde{H} / H$, see [5, Corollary 2 Section 2, No. 6 INT VII.43].

Note that $\hat{\xi}_{0}$ is a transitive space with respect to the action of $\widetilde{H}$. As in Ass. A2), we also assume that the measure $m_{0}$ is relatively $\widetilde{H}$-invariant with character $\widetilde{\gamma}$. Note that

$$
\widetilde{\gamma}(h)=\gamma(h), \quad h \in H .
$$

Finally, strengthening the analogous of A3) for $\widetilde{\Xi}$, we suppose that $\sigma$ is such that

$$
\widetilde{\gamma}\left(\sigma\left(\xi^{\prime}\right)^{-1} \sigma(\xi)\right)=1
$$

for every $\xi, \xi^{\prime} \in \Xi$ with $j(\xi)=j\left(\xi^{\prime}\right)$.

The main result of this work reads as follows.

Theorem 3 Under the above assumptions, if $\hat{\pi}$ is irreducible, then $H=\widetilde{H}$ and the map $\xi \mapsto \hat{\xi}$ is injective.

The rest of this section is devoted to the proof of this result.

To prove our main result we recall the following disintegration formula. We adopt the notation of [5, Definition 1, Section 2, No. 2 INTVII.31]. Given a character $\beta$ of $G$ and a closed subgroup $G_{0}$ of $G$, we denote by $\beta \cdot \mu_{G} / \mu_{G_{0}}$ the unique measure on the quotient space $G / G_{0}$ such that for all compactly supported continuous functions $f: G \rightarrow \mathbb{C}$

$$
\int_{G} \beta(g) f(g) \mathrm{d} \mu_{G}(g)=\int_{G / G_{0}}\left(\int_{G_{0}} f(g h) \mathrm{d} \mu_{G_{0}}(h)\right) \mathrm{d}\left(\beta \cdot \mu_{G} / \mu_{G_{0}}\right)\left(g G_{0}\right) .
$$

Observe first that, according to [5, Theorem 3, Section 2, No. 6 INT VII.43], the relatively invariant measures $\mathrm{d} \widetilde{\xi}$ and $\mathrm{d} \xi$ are proportional to $\left(\beta \cdot \mu_{G}\right) / \mu_{\widetilde{H}}$ and $(\beta$. $\left.\mu_{G}\right) / \mu_{H}$, respectively. Furthermore, note that the map

$$
(G, \widetilde{H}) \ni(g, h) \rightarrow g h \in G
$$


defines a continuous and proper right action of $\widetilde{H}$ onto $G$. The measure $\beta \cdot \mu_{G}$ is right relatively $\widetilde{H}$-invariant with character $\Delta_{\widetilde{H}}$. Then by $[5$, Corollary 1 , Section 2 , No. 8 INT VII.45], there exists a positive constant $C>0$ such that, for any $f \in L^{1}(\Xi, d \xi)$

$$
\int_{\Xi} f(\xi) \mathrm{d} \xi=C \int_{\Xi}\left(\int_{\widetilde{H} / H} f\left(\widetilde{\sigma}(\widetilde{\xi}) h \cdot \xi_{0}\right) \mathrm{d} \omega(h H)\right) \mathrm{d} \widetilde{\xi}
$$

where $\widetilde{\sigma}: \widetilde{\Xi} \rightarrow G$ is a section and the value $f\left(\widetilde{\sigma}(\widetilde{\xi}) h \cdot \xi_{0}\right)$ depends only on the left coset $h H$ since $H$ is the stability subgroup at $\xi_{0}$. The right hand side is well defined since there is a negligible set $\widetilde{E} \subset \widetilde{\Xi}$ such that if $\widetilde{\xi} \notin \widetilde{E}$, the map

$$
\widetilde{H} / H \ni h H \rightarrow f\left(\widetilde{\sigma}(\widetilde{\xi}) h . \xi_{0}\right) \in \mathbb{C}
$$

is integrable with respect to $\omega$, and the almost everywhere defined function

$$
\widetilde{\Xi} \ni \widetilde{\xi} \mapsto \int_{\widetilde{H} / H} f\left(\widetilde{\sigma}(\widetilde{\xi}) h \cdot \xi_{0}\right) \in \mathbb{C}
$$

is integrable with respect to $\mathrm{d} \widetilde{\xi}$. Furthermore, (20) is equivalent to

$$
\int_{\Xi} f(\xi) \mathrm{d} \xi=C \int_{\Xi}\left(\int_{j(\xi)=\widetilde{\xi}} f(\xi) d v_{\widetilde{\xi}}(\xi)\right) \mathrm{d} \widetilde{\xi}
$$

where $v_{\widetilde{\xi}}$ is the image measure of $\omega$ under the map

$$
\widetilde{H} / H \ni h H \mapsto \widetilde{\sigma}(\widetilde{\xi}) h . \xi_{0} \in \Xi,
$$

which is a homeomorphism from $\widetilde{H} / H$ onto the closed subset $j^{-1}(\widetilde{\xi})$. In particular, it holds true that the support of each $v_{\widetilde{\xi}}$ is $j^{-1}(\widetilde{\xi})$. As a consequence, a subset $\widetilde{E} \subset \widetilde{\Xi}$ is negligible with respect to $\mathrm{d} \widetilde{\xi}$ if and only if $j^{-1}(\widetilde{E})$ is negligible with respect to $\mathrm{d} \xi$.

The next lemma shows that the Radon transform $\mathcal{R} f(\xi)$ depends only on $j(\xi)$.

Lemma 3 For all $f \in \mathcal{A}$, there exists $\widetilde{F}: \widetilde{\Xi} \rightarrow \mathbb{C}$ and a negligible set $\widetilde{E} \subseteq \widetilde{\Xi}$ such that $j^{-1}(\widetilde{E})$ is negligible and

$$
\mathcal{R} f(\xi)=\widetilde{F}(j(\xi)), \quad \xi \notin j^{-1}(\widetilde{E}) .
$$

Furthermore, for every $\xi_{1}, \xi_{2} \in \Xi$

$$
\hat{\xi}_{1}=\hat{\xi}_{2} \quad \Longrightarrow \quad \mathcal{R} f\left(\xi_{1}\right)=\mathcal{R} f\left(\xi_{2}\right) .
$$

Proof Given $f \in \mathcal{A}$, define

$$
\left.E=\{\xi \in \Xi: f(\sigma(\xi))[\cdot]) \notin L^{1}\left(\hat{\xi}_{0}, m_{0}\right)\right\} .
$$


By (7a $)$, the set $E$ is negligible. For any two points $\xi, \xi^{\prime} \in \Xi$ such that $j(\xi)=j\left(\xi^{\prime}\right)$, taking into account that $\sigma\left(\xi^{\prime}\right)^{-1} \sigma(\xi) \in \widetilde{H}$ and by assumption (19), it holds that

$$
\begin{aligned}
\mathcal{R} f\left(\xi^{\prime}\right) & =\int_{\hat{\xi}_{0}} f\left(\sigma(\xi) \sigma(\xi)^{-1} \sigma\left(\xi^{\prime}\right)[x]\right) \mathrm{d} m_{0}(x) \\
& \left.=\widetilde{\gamma}\left(\sigma\left(\xi^{\prime}\right)^{-1} \sigma(\xi)\right) \int_{\hat{\xi}_{0}} f(\sigma(\xi))[x]\right) \mathrm{d} m_{0}(x) \\
& =\widetilde{\gamma}\left(\sigma\left(\xi^{\prime}\right)^{-1} \sigma(\xi)\right) \mathcal{R} f(\xi) \\
& =\mathcal{R} f(\xi)
\end{aligned}
$$

so that either $\xi, \xi^{\prime} \notin E$ or $\xi, \xi^{\prime} \in E$ and the claim follows with $\widetilde{E}=j(E)$ because $E=j^{-1}(j(E))$. Since $E$ is negligible, so is $\widetilde{E}$ as a consequence of [21], as already observed.

The last part immediately follows from Corollary 1.

As shown by the following corollary, $F$ is the Radon transform of $f$ associated to the pair $(X, \widetilde{\Xi})$, which trivially satisfies A1) to A4), whereas the definition of the Radon trasform requires only (7a).

Corollary 2 Let $q: \widetilde{\Xi} \rightarrow \Xi$ be a measurable section such that $q\left(\widetilde{\xi_{0}}\right)=\xi_{0}$. Then $\widetilde{\sigma}=\sigma \circ q: \widetilde{\Xi} \rightarrow G$ is a measurable section. Furthermore, the pair $(X, \widetilde{\Xi})$ satisfies A3) and (7a) for all $f \in \mathcal{A}$ and, denoting the corresponding Radon transform by $\widetilde{\mathcal{R}}$, for all $f \in \mathcal{A}$,

$$
\mathcal{R} f(\xi)=\widetilde{\mathcal{R}} f(j(\xi)) \quad \text { a.e. } \xi \in \Xi
$$

and

$$
\mathcal{R} f(q(\widetilde{\xi}))=\widetilde{\mathcal{R}} f(\widetilde{\xi}) \quad \text { a.e. } \widetilde{\xi} \in \widetilde{\Xi} .
$$

Proof Let $p: G \rightarrow \Xi$ and $\widetilde{p}: G \rightarrow \widetilde{\Xi}$ be the canonical projections, then $\widetilde{p}=j \circ p$. We readily derive

$$
\widetilde{p} \circ \widetilde{\sigma}=j \circ(p \circ \sigma) \circ q=j \circ q=\mathrm{Id}, \quad \widetilde{\sigma}\left(j\left(\xi_{0}\right)\right)=\sigma\left(\xi_{0}\right)=e,
$$

so that $\widetilde{\sigma}$ is a measurable section from $\widetilde{\Xi}$ to $G$.

From (6), with $\xi=q(\widetilde{\xi})$ and $g \in G$ we get

$$
\begin{aligned}
\iota(g) & =\gamma\left(\sigma(q(\widetilde{\xi}))^{-1} g \sigma\left(g^{-1} \cdot q(\widetilde{\xi})\right)\right) \\
& =\widetilde{\gamma}\left(\widetilde{\sigma}(\widetilde{\xi})^{-1} g\left(\widetilde{\sigma}\left(g^{-1} \cdot \widetilde{\xi}\right) \sigma\left(\xi^{\prime}\right)^{-1}\right) \sigma\left(\xi^{\prime \prime}\right)\right) \\
& =\widetilde{\gamma}\left(\widetilde{\sigma}(\widetilde{\xi})^{-1} g \widetilde{\sigma}\left(g^{-1} \cdot \widetilde{\xi}\right)\right) \widetilde{\gamma}\left(\sigma\left(\xi^{\prime}\right)^{-1} \sigma\left(\xi^{\prime \prime}\right)\right) \\
& =\widetilde{\gamma}\left(\widetilde{\sigma}(\widetilde{\xi})^{-1} g \widetilde{\sigma}\left(g^{-1} \cdot \widetilde{\xi}\right)\right)
\end{aligned}
$$

where $\xi^{\prime}=q\left(g^{-1} \cdot \widetilde{\xi}\right)$ and $\xi^{\prime \prime}=g^{-1} \cdot q(\widetilde{\xi})$ are such that $j\left(\xi^{\prime}\right)=j\left(\xi^{\prime \prime}\right)$ so that the last equality is a consequence of (19). Hence, Assumption A3) holds true. 
We now prove $(7 \mathrm{a})$ for $\widetilde{\sigma}$. Take $f \in \mathcal{A}$. Let $\widetilde{E} \subseteq \widetilde{\Xi}$ be the negligible set given by Lemma 3 Note that if $\widetilde{\xi} \notin \widetilde{E}$ then $q(\widetilde{\xi}) \notin j^{-1}(\widetilde{E})$. Thus, for $\widetilde{\xi} \notin \widetilde{E}$ we have

$$
\left.\int_{\hat{\xi}_{0}} \mid f(\widetilde{\sigma}(\widetilde{\xi}))[x]\right)\left|\mathrm{d} m_{0}(x)=\int_{\hat{\xi}_{0}}\right| f(\sigma(q(\widetilde{\xi}))[x]) \mid \mathrm{d} m_{0}(x)<+\infty,
$$

and so $f(\widetilde{\sigma}(\widetilde{\xi})[\cdot]) \in L^{1}\left(\hat{\xi}_{0}, m_{0}\right)$. Similarly, for $\widetilde{\xi} \notin \widetilde{E}$ we have

$$
\widetilde{\mathcal{R}} f(\widetilde{\xi}):=\int_{\hat{\tilde{\xi}}_{0}} f(\widetilde{\sigma}(\widetilde{\xi})[x]) \mathrm{d} m_{0}(x)=\int_{\hat{\xi}_{0}} f(\sigma(q(\widetilde{\xi}))[x]) \mathrm{d} m_{0}(x)=\mathcal{R} f(q(\widetilde{\xi})) .
$$

Finally, for $\widetilde{\xi}=j(\xi)$ with $\xi \notin j^{-1}(\widetilde{E})$ we have $j(q(\widetilde{\xi}))=\widetilde{\xi}$, and so Lemma 3 yields

$$
\widetilde{\mathcal{R}} f(j(\xi))=\mathcal{R} f(\xi),
$$

as desired.

Lemma 4 The space

$L^{2}(\Xi, \mathrm{d} \xi)_{0}=\left\{F \in L^{2}(\Xi, \mathrm{d} \xi) \mid F(\xi)=\widetilde{F}(j(\xi))\right.$ for a.e. $\xi \in \Xi$ for some $\left.\widetilde{F}: \widetilde{\Xi} \rightarrow \mathbb{C}\right\}$

is a closed $\hat{\pi}$-invariant subspace. Hence, if $\hat{\pi}$ is irreducible, then

$$
L^{2}(\Xi, \mathrm{d} \xi)_{0}=L^{2}(\Xi, \mathrm{d} \xi)
$$

Proof We first observe that, given $F \in L^{2}(\Xi, \mathrm{d} \xi)_{0}$, by construction there exists $\widetilde{F}: \widetilde{\Xi} \rightarrow \mathbb{C}$ such that $F$ and $\widetilde{F} \circ j$ are equal almost everywhere. Hence, we can always assume that $F=\widetilde{F} \circ j$.

Let $\left(F_{n}\right)$ be a sequence in $L^{2}(\Xi, \mathrm{d} \xi)_{0}$ converging to $F \in L^{2}(\Xi, \mathrm{d} \xi)$. As observed, we can assume that $F_{n}=\widetilde{F}_{n} \circ j$ where $\widetilde{F}_{n}: \widetilde{\Xi} \rightarrow \mathbb{C}$. Since $F_{n}$ converges to $F$, possibly passing to a subsequence, there exists a negligible set $E$ such that

$$
\lim _{n \rightarrow+\infty} F_{n}(\xi)=\lim _{n \rightarrow+\infty} \widetilde{F}_{n}(j(\xi))=F(\xi), \quad \xi \notin E .
$$

Define $\widetilde{F}: \widetilde{\Xi} \rightarrow \mathbb{C}$ as

$$
\widetilde{F}(\widetilde{\xi})= \begin{cases}\lim _{n \rightarrow+\infty} \widetilde{F}_{n}(\widetilde{\xi}) & \widetilde{\xi} \in j(\Xi \backslash E), \\ 0 & \widetilde{\xi} \notin j(\Xi \backslash E) .\end{cases}
$$

Then by construction

$$
F(\xi)=\widetilde{F}(j(\xi)), \quad \xi \notin E .
$$

It follows that $L^{2}(\Xi, \mathrm{d} \xi)_{0}$ is closed. We now prove that it is $\hat{\pi}$-invariant. Given $g \in G$, for all $F \in L^{2}(\Xi, \mathrm{d} \xi)_{0}$ 


$$
\begin{aligned}
\hat{\pi}(g) F(\xi) & =\beta(g)^{-1 / 2} F\left(g^{-1} \cdot \xi\right) \\
& =\beta(g)^{-1 / 2} \widetilde{F}\left(j\left(g^{-1} \cdot \xi\right)\right) \\
& =\beta(g)^{-1 / 2} \widetilde{F}\left(g^{-1} \cdot j(\xi)\right),
\end{aligned}
$$

so that $L^{2}(\Xi, \mathrm{d} \xi)_{0}$ is $\hat{\pi}$-invariant.

Assume that $\hat{\pi}$ is irreducible, then $L^{2}(\Xi, \mathrm{d} \xi)_{0}$ is zero or the full space. Since $\mathcal{A}$ is not trivial, there exists a non-zero $f \in \mathcal{A}$ such that $\mathcal{R} f \in L^{2}(\Xi, \mathrm{d} \xi)_{0}$ by (22). Furthermore, $\mathcal{R} f \neq 0$ since $\mathcal{I} \mathcal{R} f=Q f \neq 0$ because $Q$ is an isometry. Hence $L^{2}(\Xi, \mathrm{d} \xi)_{0}$ is non-trivial and $L^{2}(\Xi, \mathrm{d} \xi)_{0}=L^{2}(\Xi, \mathrm{d} \xi)$.

The proof of Theorem 3 will be an immediate consequence of Proposition 1 and of the following result.

Proposition 2 Assume that $\widetilde{H} \neq H$, then $\hat{\pi}$ is not irreducible.

Proof Suppose by contradiction that $\hat{\pi}$ is irreducible, then $L^{2}(\Xi, \mathrm{d} \xi)_{0}=L^{2}(\Xi, \mathrm{d} \xi)$ by Lemma4

We first prove that $\omega$ is a finite measure. Fix $f \in L^{1}(\Xi, \mathrm{d} \xi) \cap L^{2}(\Xi, \mathrm{d} \xi)$ such that $f$ is positive and $f \neq 0$, then there exists $F: \widetilde{\Xi} \rightarrow \mathbb{C}$ such that $F(j(\xi))=f(\xi)$ for all $\xi \notin E$ where $E \subset \Xi$ is negligible. Hence, by (21)

$$
\begin{aligned}
0<\int_{\Xi} f(\xi) \mathrm{d} \xi & =\int_{\Xi} F(j(\xi)) \mathrm{d} \xi=C \int_{\widetilde{\Xi}}\left(\int_{j(\xi)=\widetilde{\xi}} F(j(\xi)) \mathrm{d} v_{\widetilde{\xi}}(\xi)\right) d \widetilde{\xi} \\
& =C \int_{\widetilde{\Xi}} F(\widetilde{\xi}) v_{\widetilde{\xi}}(\Xi) \mathrm{d} \widetilde{\xi}<\infty .
\end{aligned}
$$

It follows that there exists a negligible subset $\widetilde{E} \subset \widetilde{\Xi}$ such that for all $\widetilde{\xi} \notin \widetilde{E}, v_{\widetilde{\xi}}(\Xi)$ is finite. By construction of $v_{\widetilde{\xi}}$ as image measure

$$
v_{\widetilde{\xi}}(\Xi)=\omega(\widetilde{H} / H)<+\infty, \quad \widetilde{\xi} \in \widetilde{\Xi} .
$$

For every $\widetilde{\xi} \in \widetilde{\Xi}$, the map $h H \mapsto(\widetilde{\sigma}(\widetilde{\xi}) h) . \xi_{0}$ is a homeomorphism from $\widetilde{H} / H$ onto $j^{-1}(\widetilde{\xi})$. Thus, since $\widetilde{H} / H$ is not a singleton, there exist $\xi_{1}, \xi_{2} \in j^{-1}(\widetilde{\xi})$ such that $\xi_{1} \neq \xi_{2}$ and, hence, two disjoint compact neighbourhoods $V_{\widetilde{\xi}}^{1}, V_{\widetilde{\xi}}^{2}$ of $\xi_{1}$ and $\xi_{2}$, respectively (see Figure 8). Since the support of $v_{\widetilde{\xi}}$ is $j^{-1}(\widetilde{\xi})$, then

$$
v_{\widetilde{\xi}}\left(V_{\widetilde{\xi}}^{1}\right)>0, \quad v_{\widetilde{\xi}}\left(V_{\widetilde{\xi}}^{2}\right)>0
$$

Let now $K \subset \widetilde{\Xi}$ be a compact set of positive measure,

$$
Z=\left\{\xi \in \Xi: j(\xi) \in K, \xi \in V_{j(\xi)}^{1}\right\},
$$

and $f$ be the corresponding characteristic function of $Z$. By applying (21) we obtain 


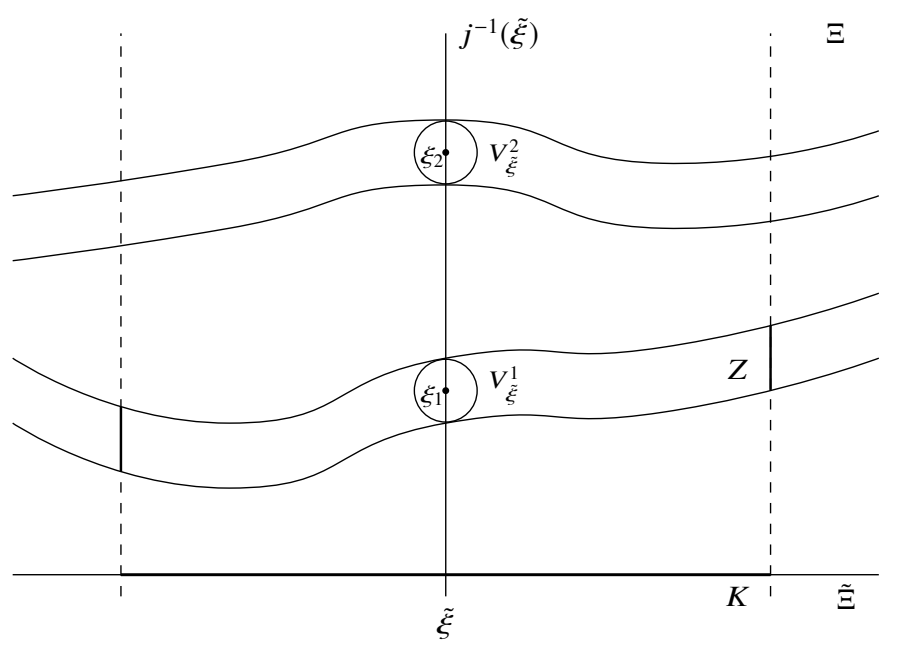

Fig. 1 The setup considered in the proof of Proposition 2

$$
0<\int_{\Xi}|f(\xi)|^{2} \mathrm{~d} \xi=\int_{\Xi} f(\xi) \mathrm{d} \xi=C \int_{K} v_{\widetilde{\xi}}\left(V_{\widetilde{\xi}}^{1}\right) \mathrm{d} \widetilde{\xi} \leq C \omega(\widetilde{H} / H) \int_{K} \mathrm{~d} \widetilde{\xi}<+\infty,
$$

which is finite since $\omega(\widetilde{H} / H)<+\infty$ and $K$ is compact. We can apply 21) since $f$ is positive [5, item c), Corollary 1, Section 2, No. 8 INT VII.45]. Hence $f \in L^{2}(\Xi, \mathrm{d} \xi)$ and, as above, there exists $F: \widetilde{\Xi} \rightarrow \mathbb{C}$ such that $F(j(\xi))=f(\xi)$ for all $\xi \notin E^{\prime}$ where $E^{\prime} \subset \Xi$ is negligible. Since $E^{\prime}$ is negligible, by (21) applied to the characteristic function of $E^{\prime}$ there exists a negligible subset $\widetilde{E^{\prime}} \subset \widetilde{\Xi}$ such that

$$
v_{\widetilde{\xi}}\left(E^{\prime} \cap j^{-1}(\widetilde{\xi})\right)=0, \quad \widetilde{\xi} \notin \widetilde{E^{\prime}} .
$$

Choose an arbitrary $\widetilde{\xi} \in K \backslash \widetilde{E}^{\prime}$. By (24) and (25), for $i=1,2$ there exists $\xi^{i} \in V_{\widetilde{\xi}}^{i} \backslash E^{\prime}$ such that $j\left(\xi^{i}\right)=\widetilde{E}^{\prime}$. Thus

$$
F(\widetilde{\xi})=F\left(j\left(\xi^{i}\right)\right)=f\left(\xi^{i}\right)= \begin{cases}1 & \text { if } i=1 \\ 0 & \text { if } i=2\end{cases}
$$

which is absurd. 


\section{3D-signals: Radon and ray transforms}

\subsection{The Radon transform on $\mathbb{R}^{3}$}

\subsubsection{Groups and spaces}

The Radon transform on $\mathbb{R}^{3}$ of a signal $f$ is defined as the integral of $f$ over the set of planes in $\mathbb{R}^{3}$. We show that this is an example of our construction.

The input space is $X=\mathbb{R}^{3}$ and the group is $\operatorname{SIM}(3)$, the semi-direct product $\mathbb{R}^{3} \rtimes K$, with $K=\left\{a R \in \mathrm{GL}(3, \mathbb{R}): R \in \mathrm{SO}(3), a \in \mathbb{R}_{+}\right\}$. Under the identification $K \simeq \operatorname{SO}(3) \times \mathbb{R}_{+}$, we write $(\mathbf{b}, R, a)$ for the elements in $\operatorname{SIM}(3)$ and the group law becomes

$$
(\mathbf{b}, R, a)\left(\mathbf{b}^{\prime}, R^{\prime}, a^{\prime}\right)=\left(\mathbf{b}+a R \mathbf{b}^{\prime}, R R^{\prime}, a a^{\prime}\right) .
$$

A left Haar measure of $\operatorname{SIM}(3)$ is given by

$$
\mathrm{d} \mu(\mathbf{b}, R, a)=a^{-4} \mathrm{~d} \mathbf{b d} R \mathrm{~d} a,
$$

where $\mathrm{db}$ and $\mathrm{d} a$ are the Lebesgue measures on $\mathbb{R}^{3}$ and $\mathbb{R}_{+}$, respectively, and $\mathrm{d} R$ is a Haar measure of $\operatorname{SO}(3)$. The group $\operatorname{SIM}(3)$ acts on $\mathbb{R}^{3}$ by the canonical action

$$
(\mathbf{b}, R, a)[\mathbf{x}]=\mathbf{b}+a R \mathbf{x}, \quad(\mathbf{b}, R, a) \in \operatorname{SIM}(3), \mathbf{x} \in \mathbb{R}^{3} .
$$

The isotropy at the origin $\mathbf{x}_{0}=0$ is the subgroup $\{(0, k): k \in K\}$ which we identify with $K$, so that $X \simeq \operatorname{SIM}(3) / K$. Furthermore, the Lebesgue measure $\mathrm{d} \mathbf{x}$ on $\mathbb{R}^{3}$ is a relatively $\operatorname{SIM}(3)$-invariant measure with positive character $\alpha(\mathbf{b}, R, a)=a^{3}$. It remains to choose the closed subgroup $H$ of $\operatorname{SIM}(3)$ in such a way that $\{\widehat{\xi}\}$ is the set of planes in $\mathbb{R}^{3}$. We consider $H=\left(\mathbb{R}^{2} \times\{0\}\right) \rtimes\left(\mathrm{O}(2) \times \mathbb{R}_{+}\right)$, where $\mathrm{O}(2)$ denotes the subgroup of rotations leaving the plane $z=0$ invariant, i.e. it consists of the matrices of the form

$$
R=\left[\begin{array}{cc}
R_{ \pm} R_{\theta} & 0 \\
0 & \pm 1
\end{array}\right]
$$

where $R_{\theta} \in \mathrm{SO}(2), R_{+}$is the identity and $R_{-}=\left[\begin{array}{cc}1 & 0 \\ 0 & -1\end{array}\right]$. By (3), the root manifold is the $x y$-plane

$$
\hat{\xi_{0}}=H\left[\mathbf{x}_{0}\right]=\left\{\mathbf{x} \in \mathbb{R}^{3}: \mathbf{x} \cdot \mathbf{e}_{3}=0\right\}
$$

and it is easy to verify that $m_{0}=\mathrm{d} x \mathrm{~d} y$ is a relatively $H$-invariant measure on $\hat{\xi}_{0}$ with character $\gamma(\mathbf{b}, R, a)=a^{2}$. Furthermore, for each $\xi=(\mathbf{b}, R, a) H \in \Xi=\operatorname{SIM}(3) / H$, by (4) we compute

$$
\hat{\xi}=(\mathbf{b}, R, a)\left[\hat{\xi}_{0}\right]=\left\{\mathbf{x} \in \mathbb{R}^{3}: R_{\mathbf{e}_{3}} \cdot \mathbf{x}=R \mathbf{e}_{3} \cdot \mathbf{b}\right\},
$$

which is the plane perpendicular to the vector $R \mathbf{e}_{3}$ and passing through $\mathbf{b}$. It is worth observing that $H$ is the maximal closed subgroup of $\operatorname{SIM}(3)$ which satisfies $h\left[\hat{\xi}_{0}\right]=\hat{\xi}_{0}$ for every $h \in H$ and then, by Proposition 1 , the map $\xi \rightarrow \hat{\xi}$ is injective. Had we chosen $H^{\prime}=\left(\mathbb{R}^{2} \times\{0\}\right) \rtimes\left(\mathrm{SO}(2) \times \mathbb{R}_{+}\right)$, we would have had $H^{\prime} \subsetneq \widetilde{H}$ and, 
indeed, the map $\xi^{\prime} \mapsto \widehat{\xi^{\prime}}$ would not have been injective, since every plane would have been labeled by two different $\xi^{\prime}$.

We identify the coset space $\Xi=\operatorname{SIM}(3) / H$ with the set $[0, \pi)_{*}^{2} \times \mathbb{R}$, where

$$
[0, \pi)_{*}^{2}=([0, \pi) \times(0, \pi)) \cup\{(0,0)\}
$$

and we write

$$
\mathbf{n}(\theta, \varphi)={ }^{t}(\sin \varphi \cos \theta, \sin \varphi \sin \theta, \cos \varphi)
$$

for every $(\theta, \varphi) \in[0, \pi)_{*}^{2}$. The group $\operatorname{SIM}(3)$ acts on $[0, \pi)_{*}^{2} \times \mathbb{R}$ by the transitive action

$$
(\mathbf{b}, R, a) .(\theta, \varphi, t)=\left(\theta_{R}, \varphi_{R}, R \mathbf{n}(\theta, \varphi) \cdot \mathbf{n}\left(\theta_{R}, \varphi_{R}\right)(a t+R \mathbf{n}(\theta, \varphi) \cdot \mathbf{b})\right),
$$

where $\left(\theta_{R}, \varphi_{R}\right) \in[0, \pi)_{*}^{2}$ is such that $R \mathbf{n}(\theta, \varphi)= \pm \mathbf{n}\left(\theta_{R}, \varphi_{R}\right)$. Since the stability subgroup at $(0,0,0)$ is $H$, then $\operatorname{SIM}(3) / H \simeq[0, \pi)_{*}^{2} \times \mathbb{R}$ under the canonical isomorphism $(\mathbf{b}, R, a) H \mapsto(\mathbf{b}, R, a) .(0,0,0)$.

We endow $\Xi$ with the measure $\mathrm{d} \xi=\sin \varphi \mathrm{d} \theta \mathrm{d} \varphi \mathrm{d} t$, where $\mathrm{d} \theta, \mathrm{d} \varphi$ and $\mathrm{d} t$ are the Lebesgue measure on $[0, \pi)$ and $\mathbb{R}$, respectively. It is easy to verify that $\mathrm{d} \xi$ is a

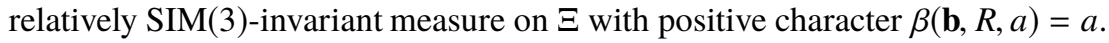

\subsubsection{The representations}

The group $\operatorname{SIM}(3)$ acts on $L^{2}\left(\mathbb{R}^{3}\right)$ by means of the unitary representation $\pi$ defined by

$$
\pi(\mathbf{b}, R, a) f(\mathbf{x})=a^{-\frac{3}{2}} f\left(a^{-1} R^{-1}(\mathbf{x}-\mathbf{b})\right),
$$

The dual action $\mathbb{R}^{3} \times K \ni(\eta, k) \mapsto{ }^{t} k \eta$ has a single open orbit $O=\mathbb{R}^{3}$ for $\mathbf{e}_{3}$ of full measure and the stabilizer $K_{\mathbf{e}_{3}} \simeq \mathrm{SO}(2) \times\{1\}$ is compact. Then, the representation $\pi$ is irreducible and square-integrable, see [2].

Furthermore, the quasi-regular representation $\hat{\pi}$ of $\operatorname{SIM}(3)$ acting on $L^{2}(\Xi, \mathrm{d} \xi)$ as

$$
\hat{\pi}(\mathbf{b}, R, a) F(\theta, \varphi, t)=a^{-\frac{1}{2}} F\left(\theta_{R^{-1}}, \varphi_{R^{-1}}, R^{-1} \mathbf{n}(\theta, \varphi) \cdot \mathbf{n}\left(\theta_{R^{-1}}, \varphi_{R^{-1}}\right) \frac{t-\mathbf{n}(\theta, \varphi) \cdot \mathbf{b}}{a}\right),
$$

is irreducible, too. As a consequence, Theorem 3 guarantees that the map $\xi \mapsto \hat{\xi}$ is injective. Let us consider again the situation with the choice $H^{\prime}=\left(\mathbb{R}^{2} \times\{0\}\right) \rtimes$ $\left(\mathrm{SO}(2) \times \mathbb{R}_{+}\right)$. In this case, $\Xi^{\prime}=\operatorname{SIM}(3) / \mathrm{H}^{\prime}$ may be identified with $S^{2} \times \mathbb{R}$, and we have already observed that the map

$$
\xi^{\prime}=(\mathbf{n}, t) \mapsto \hat{\xi}_{\mathbf{n}, t}=\left\{\mathbf{x} \in \mathbb{R}^{3}: \mathbf{n} \cdot \mathbf{x}=t\right\}
$$

is not injective, since $\left(\mathbf{n}_{1}, t_{1}\right)$ and $\left(\mathbf{n}_{2}, t_{2}\right)$ identify the same plane if

$$
\left(\mathbf{n}_{1}, t_{1}\right)= \pm\left(\mathbf{n}_{2}, t_{2}\right) .
$$


In the notation of Section 3, this corresponds to $j\left(\mathbf{n}_{1}, t_{1}\right)=j\left(\mathbf{n}_{2}, t_{2}\right)$. According to Theorem 3 , this implies that the corresponding quasi-regular representation $\widetilde{\pi^{\prime}}$ cannot be irreducible. Let us verify this explicitly, in order to visualise the link between the irreducibility of $\widehat{\pi^{\prime}}$ and the injectivity of $\xi^{\prime} \mapsto \widehat{\xi}^{\prime}$ in this example. By arguing as above, it is easy to prove that

$$
\widehat{\pi^{\prime}}(\mathbf{b}, R, a) F(\mathbf{n}, t)=a^{-\frac{1}{2}} F\left(R^{-1} \mathbf{n}, \frac{t-\mathbf{n} \cdot \mathbf{b}}{a}\right) .
$$

Thus, using the notation of Lemma 4 the set

$$
L^{2}\left(\Xi^{\prime}, d \xi^{\prime}\right)_{0}=\left\{F \in L^{2}\left(\Xi^{\prime}, d \xi^{\prime}\right): F\left(\mathbf{n}_{1}, t_{1}\right)=F\left(\mathbf{n}_{2}, t_{2}\right) \text { if }(28) \text { holds }\right\}
$$

is a closed $\widehat{\pi^{\prime}}$-invariant proper subspace of $L^{2}\left(\Xi^{\prime}, d \xi^{\prime}\right)$. Hence, $\widehat{\pi^{\prime}}$ is not irreducible.

\subsubsection{The Radon transform}

In order to define the Radon transform we need to endow each $\hat{\xi}$ with a suitable measure. Since the measure $m_{0}$ is $H$-relatively invariant, the choice of the representative of $\xi$ is crucial. We fix the Borel section

$$
\sigma: \Xi \rightarrow G, \quad \sigma(\theta, \varphi, t)=\left(t \mathbf{n}(\theta, \varphi), R_{\theta, \varphi}, 1\right),
$$

with $R_{\theta, \varphi} \in \mathrm{SO}(3)$ such that $R_{\theta, \varphi} \mathbf{e}_{3}=\mathbf{n}(\theta, \varphi)$. We observe that, since $\gamma$ extends to a positive character of $G$, assumption (A4) is implied by the stronger condition $\gamma(\sigma(\xi))=1$ for every $\xi \in \Xi$. Then, we compute the Radon transform by (9) obtaining

$$
\mathcal{R} f(\theta, \varphi, t)=\int_{\mathbb{R}^{2}} f\left(t \mathbf{n}(\theta, \varphi)+R_{\theta, \varphi}(x, y, 0)\right) \mathrm{d} x \mathrm{~d} y,
$$

which is the integral of $f$ on the plane of equation $\mathbf{n}(\theta, \varphi) \cdot \mathbf{x}=t$. As a consequence of Fubini theorem, equation (29) makes sense for instance if $f \in L^{1}\left(\mathbb{R}^{3}\right)$.

We recall a crucial result in Radon transform theory in its standard version, known as Fourier slice theorem. We denote by I the identity operator.

Proposition 3 For every $f \in L^{1}\left(\mathbb{R}^{3}\right)$

$$
(I \otimes \mathcal{F}) \mathcal{R} f(\theta, \varphi, \tau)=\mathcal{F} f(\tau \mathbf{n}(\theta, \varphi)),
$$

for all $(\theta, \varphi, \tau) \in[0, \pi)_{*}^{2} \times \mathbb{R}$.

Here the Fourier transform on the right-hand side is in $\mathbb{R}^{3}$, whereas the operator $\mathcal{F}$ on the left-hand side is one-dimensional and acts on the variabile $t$. We repeat this slight abuse of notation in other formulas below.

We show that assumption A6) holds true. Let $S^{2}$ be the sphere in $\mathbb{R}^{3}$ and denote by $\mathcal{S}\left(\mathbb{R}^{3}\right)$ and $\mathcal{S}\left(S^{2} \times \mathbb{R}\right)$ the Schwartz spaces of rapidly decreasing functions on $\mathbb{R}^{3}$ 
and on $S^{2} \times \mathbb{R}$, respectively, and by $\mathcal{S}^{\prime}\left(\mathbb{R}^{3}\right)$ and $\mathcal{S}^{\prime}\left(S^{2} \times \mathbb{R}\right)$ the corresponding spaces of tempered distributions; see [13, Chapter 1.2] for the definition on $S^{2} \times \mathbb{R}$.

We extend the Radon transform $\mathcal{R}$ as an even function on $S^{2}$ and we denote it by $\mathcal{R}_{e}$, i.e.

$$
\mathcal{R}_{e} f(\mathbf{u}, t)=\mathcal{R} f\left(\theta_{\mathbf{u}}, \varphi_{\mathbf{u}}, \mathbf{u} \cdot \mathbf{n}\left(\theta_{\mathbf{u}}, \varphi_{\mathbf{u}}\right) t\right),
$$

where $\left(\theta_{\mathbf{u}}, \varphi_{\mathbf{u}}\right) \in[0, \pi)_{*}^{2}$ is such that $\mathbf{n}\left(\theta_{\mathbf{u}}, \varphi_{\mathbf{u}}\right)= \pm \mathbf{u}$.

We recall that, since $\mathcal{R}_{e}$ is a continuous map from $\mathcal{S}\left(\mathbb{R}^{3}\right)$ into $\mathcal{S}\left(S^{2} \times \mathbb{R}\right)$ (see [11]), given $F \in \mathcal{S}^{\prime}\left(S^{2} \times \mathbb{R}\right)$, the tempered distribution $\mathcal{R}_{e}^{\#} F: \mathcal{S}\left(\mathbb{R}^{3}\right) \rightarrow \mathbb{C}$ given by

$$
\left\langle\mathcal{R}_{e}^{\#} F, f\right\rangle=\left\langle F, \mathcal{R}_{e} f\right\rangle
$$

is well-defined. If $F \in \mathcal{S}\left(S^{2} \times \mathbb{R}\right)$, by Theorem 1.4 in [18, Chapter 2], the tempered distribution $\mathcal{F} \mathcal{R}_{e}^{\#} F$ is represented by the function

$$
\mathcal{F} \mathcal{R}_{e}^{\#} F(\mathbf{v})=|\mathbf{v}|^{-2}[(I \otimes \mathcal{F}) F(\mathbf{v} /|\mathbf{v}|,|\mathbf{v}|)+(I \otimes \mathcal{F}) F(-\mathbf{v} /|\mathbf{v}|,-|\mathbf{v}|)] .
$$

By equation $31, \mathcal{R}_{e}^{\#} F$ is in $L^{2}\left(\mathbb{R}^{3}\right)$ provided that

$$
\int_{\mathbb{R}} t^{m} F(\mathbf{u}, t) \mathrm{d} t=0, \quad m \in \mathbb{N} .
$$

We fix a non-zero $F \in \mathcal{S}\left(S^{2} \times \mathbb{R}\right)$ which satisfies (32) and the symmetry condition $F(\mathbf{u}, t)=F(-\mathbf{u},-t)$ and we denote its restriction to $[0, \pi)_{*}^{2} \times \mathbb{R}$ by $F_{0}$, that is

$$
F_{0}(\theta, \varphi, t)=F(\mathbf{n}(\theta, \varphi), t),
$$

for every $(\theta, \varphi, t) \in[0, \pi)_{*}^{2} \times \mathbb{R}$. Then, there exists a positive constant $C$ such that

$$
\left|\left\langle F_{0}, \mathcal{R} f\right\rangle_{L^{2}\left([0, \pi)_{*}^{2} \times \mathbb{R}\right)}\right|=\frac{1}{2}\left|\left\langle F, \mathcal{R}_{e} f\right\rangle_{L^{2}\left(S^{2} \times \mathbb{R}\right)}\right|=\left|\left\langle\mathcal{R}_{e}^{\#} F, f\right\rangle\right| \leq C\|f\|,
$$

for any $f \in \mathcal{S}\left(\mathbb{R}^{3}\right)$. Therefore, if we take $f_{0} \in \mathcal{S}\left(\mathbb{R}^{3}\right)$ and define the vector subspace $\mathcal{A}=\operatorname{span}\left\{\pi(g) f_{0}: g \in G\right\} \subseteq \mathcal{S}\left(\mathbb{R}^{3}\right)$, then the domain of the adjoint of the restriction of $\mathcal{R}$ to $\mathcal{A}$ is non-trivial since $F_{0} \in \operatorname{dom}\left(\mathcal{R}^{*}\right)$ and assumption A6) holds true.

\subsubsection{The unitarization theorem}

By Theorem 1 the Radon transform $\mathcal{R}: \mathcal{A} \rightarrow L^{2}(\Xi, \mathrm{d} \xi)$ admits a unique closure $\overline{\mathcal{R}}$ which satisfies

$$
\overline{\mathcal{R}} \pi(\mathbf{b}, R, a)=\chi(\mathbf{b}, R, a)^{-1} \hat{\pi}(\mathbf{b}, R, a) \overline{\mathcal{R}}, \quad(\mathbf{b}, R, a) \in G,
$$

where $\chi(\mathbf{b}, R, a)=a$ since $\alpha(\mathbf{b}, R, a)=a^{3}, \beta(\mathbf{b}, R, a)=a$ and $\gamma(\mathbf{b}, R, a)=a^{2}$. Furthermore, there exists a unique positive self-adjoint operator 


$$
\mathcal{I}: \operatorname{dom}(\mathcal{I}) \supseteq \operatorname{Im} \overline{\mathcal{R}} \rightarrow L^{2}(\Xi, \mathrm{d} \xi),
$$

semi-invariant with weight $\chi(\mathbf{b}, R, a)^{-1}=a^{-1}$ with the property that the composite operator $\mathcal{I} \overline{\mathcal{R}}$ extends to a unitary operator $Q: L^{2}(X, \mathrm{~d} x) \rightarrow L^{2}(\Xi, \mathrm{d} \xi)$ intertwining $\pi$ and $\hat{\pi}$, namely

$$
\hat{\pi}(g) Q \pi(g)^{-1}=Q, \quad g \in G .
$$

We can provide an explicit formula for $\mathcal{I}$.

Consider the subspace

$$
\mathcal{D}=\left\{f \in L^{2}\left([0, \pi)_{*}^{2} \times \mathbb{R}\right): \int_{[0, \pi)_{*}^{2} \times \mathbb{R}}|\tau|^{2}|(I \otimes \mathcal{F}) f(\theta, \varphi, \tau)|^{2} \sin \varphi \mathrm{d} \theta \mathrm{d} \varphi \mathrm{d} \tau<+\infty\right\}
$$

and define the operator $\mathcal{J}: \mathcal{D} \rightarrow L^{2}\left([0, \pi)_{*}^{2} \times \mathbb{R}\right)$ by

$$
(I \otimes \mathcal{F}) \mathcal{J} f(\theta, \varphi, \tau)=|\tau|(I \otimes \mathcal{F}) f(\theta, \varphi, \tau),
$$

a Fourier multiplier with respect to the variable $t$. A direct calculation shows that $\mathcal{J}$ is a densely defined positive self-adjoint injective operator and is semi-invariant with weight $\zeta(g)=\chi(g)^{-1}=a^{-1}$. By [7, Theorem 1], there exists $c>0$ such that $\mathcal{I}=c \mathcal{J}$ and we now show that $c=1$. Take a non-zero function $f \in \mathcal{A}$. Then, by Plancherel theorem and Proposition 3 we have that

$$
\begin{aligned}
\|f\|^{2}=\|\mathcal{I} \mathcal{R} f\|_{L^{2}\left([0, \pi)_{*}^{2} \times \mathbb{R}\right)}^{2} & =c^{2}\|(I \otimes \mathcal{F}) \mathcal{J} \mathcal{R} f\|_{L^{2}\left([0, \pi)_{*}^{2} \times \mathbb{R}\right)}^{2} \\
& =c^{2} \int_{[0, \pi)_{*}^{2} \times \mathbb{R}}|(I \otimes \mathcal{F}) \mathcal{R} f(\theta, \varphi, \tau)|^{2}|\tau|^{2} \sin \varphi \mathrm{d} \theta \mathrm{d} \varphi \mathrm{d} \tau \\
& =c^{2} \int_{[0, \pi)_{*}^{2} \times \mathbb{R}}|\mathcal{F} f(\tau \mathbf{n}(\theta, \varphi))|^{2}|\tau|^{2} \sin \varphi \mathrm{d} \theta \mathrm{d} \varphi \mathrm{d} \tau \\
& =c^{2}\|f\|^{2} .
\end{aligned}
$$

Thus, we obtain $c=1$.

\subsubsection{The inversion formula}

By Theorem 2 , for any $f \in \mathcal{A}$ we have the reconstruction formula

$$
f=\int_{\operatorname{SIM}(3)} a^{-\frac{9}{2}}\langle\overline{\mathcal{R}} f, \hat{\pi}(\mathbf{b}, R, a) \Psi\rangle_{L^{2}(\Xi, \mathrm{d} \xi)} \psi\left(a^{-1} R^{-1}(\mathbf{x}-\mathbf{b})\right) \mathrm{d} \mathbf{b} \mathrm{d} R \mathrm{~d} a,
$$

where the integral is weakly convergent and where we used that $\chi(\mathbf{b}, R, a)=a$, the expression of the Haar measure of $\operatorname{SIM}(3)$ given in (26) and the expression of $\pi$ given in (27). 


\subsection{The X-ray transform.}

The X-ray transform in the Euclidean 3-space maps a function on $\mathbb{R}^{3}$ into the set of integrals over the lines and the X-ray reconstruction problem consists in reconstructing a signal $f$ by means of its line integrals.

\subsubsection{Groups and spaces}

Take the same group $G=\operatorname{SIM}(3)$ as in subsection 4.1 namely $G=\mathbb{R}^{3} \rtimes K$, with $K=\left\{a R \in \mathrm{GL}(3, \mathbb{R}): R \in \mathrm{SO}(3), a \in \mathbb{R}_{+}\right\}$. Firstly, we choose $X=\mathbb{R}^{3}$ and, for what concerns this space, we keep the notation as in subsection 4.1. Then, we consider the space $\Xi=G / H$, where $H=(\{(0,0)\} \times \mathbb{R}) \rtimes\left(\times \mathbb{R}_{+}\right)$. By (3), the root manifold is then

$$
\hat{\xi_{0}}=\left\{t \mathbf{e}_{3}: t \in \mathbb{R}\right\}
$$

and it is easy to verify that $m_{0}=\mathrm{d} t$ is a relatively $H$-invariant measure on $\hat{\xi}_{0}$ with character $\gamma(\mathbf{b}, R, a)=a$. Furthermore, for each $\xi=(\mathbf{b}, R, a) H \in \Xi$, by (4) we compute

$$
\hat{\xi}=(\mathbf{b}, R, a)\left[\hat{\xi}_{0}\right]=\left\{t \mathbf{R e}_{3}+\mathbf{b}: t \in \mathbb{R}\right\},
$$

which is the line parallel to the vector $R \mathbf{e}_{3}$ and passing through the point $\mathbf{b}$. It is worth observing that $H$ is the maximal closed subgroup of SIM(3) which satisfies $h\left[\hat{\xi}_{0}\right]=\hat{\xi}_{0}$ for every $h \in H$ and then, by Proposition 1 the map $\xi \rightarrow \hat{\xi}$ is injective.

The coset space $\Xi=\operatorname{SIM}(3) / H$ can be identified with the set $T=\{(\theta, \varphi, \mathbf{t})$ : $\left.(\theta, \varphi) \in[0, \pi)_{*}^{2}, \mathbf{t} \in(\theta, \varphi)^{\perp}\right\}$, where $(\theta, \varphi)^{\perp}$ denotes the plane passing through the origin and perpendicular to the vector $\mathbf{n}(\theta, \varphi)$, i.e. the plane of equation $\mathbf{n}(\theta, \varphi) \cdot \mathbf{x}=0$. The group $\operatorname{SIM}(3)$ acts on $T$ by the action

$$
(\mathbf{b}, R, a) .(\theta, \varphi, \mathbf{t})=\left(\theta_{R}, \varphi_{R}, \mathbf{t}+a R \mathbf{b}-\left(\mathbf{n}\left(\theta_{R}, \varphi_{R}\right) \cdot(\mathbf{t}+a R \mathbf{b})\right) \mathbf{n}\left(\theta_{R}, \varphi_{R}\right)\right),
$$

where we recall that $\left(\theta_{R}, \varphi_{R}\right) \in[0, \pi)_{*}^{2}$ is such that $R \mathbf{n}(\theta, \varphi)= \pm \mathbf{n}\left(\theta_{R}, \varphi_{R}\right)$. Since the stability subgroup at $(0,0,0)$ is $H$, then $\operatorname{SIM}(3) / H \simeq T$ under the canonical isomorphism $(\mathbf{b}, R, a) H \mapsto(\mathbf{b}, R, a) .(0,0,0)$.

We endow $\Xi$ with the measure $\mathrm{d} \xi=\sin \varphi \mathrm{d} \theta \mathrm{d} \varphi \mathrm{d} \mathbf{t}$, with $\mathrm{d} \theta, \mathrm{d} \varphi$ and dt being the Lebesgue measure on $[0, \pi)$ and $\mathbb{R}^{3}$, respectively. It is easy to verify that $\mathrm{d} \xi$ is a

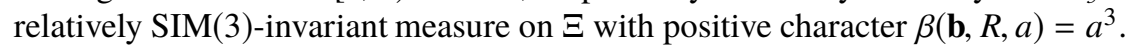

\subsubsection{The representations}

We recall that the group $\operatorname{SIM}(3)$ acts on $L^{2}\left(\mathbb{R}^{3}\right)$ by means of the unitary irreducible representation $\pi$ defined by

$$
\pi(\mathbf{b}, R, a) f(\mathbf{x})=a^{-\frac{3}{2}} f\left(a^{-1} R^{-1}(\mathbf{x}-\mathbf{b})\right)
$$


Furthermore, the quasi-regular representation $\hat{\pi}$ of $\operatorname{SIM}(3)$ acting on $L^{2}(\Xi, \mathrm{d} \xi)$ as

$$
\begin{aligned}
& \hat{\pi}(\mathbf{b}, R, a) F(\theta, \varphi, \mathbf{t})= \\
& a^{-\frac{3}{2}} F\left(\theta_{R^{-1}}, \varphi_{R^{-1}}, a^{-1} R^{-1}(\mathbf{t}-\mathbf{b})-\left(\mathbf{n}\left(\theta_{R^{-1}}, \varphi_{R^{-1}}\right) \cdot a^{-1} R^{-1}(\mathbf{t}-\mathbf{b})\right) \mathbf{n}\left(\theta_{R^{-1}}, \varphi_{R^{-1}}\right)\right),
\end{aligned}
$$

is irreducible, too.

\subsubsection{The Radon transform}

We fix a Borel section

$$
\sigma: \Xi \rightarrow G, \quad \sigma(\theta, \varphi, \mathbf{t})=\left(\mathbf{t}, R_{\theta, \varphi}, 1\right),
$$

with $R_{\theta, \varphi} \in S O(3)$ such that $R_{\theta, \varphi} \mathbf{e}_{3}=\mathbf{n}(\theta, \varphi)$. We observe that, since $\gamma$ extends to a positive character of $G$, assumption (A4) is implied by the stronger condition $\gamma(\sigma(\xi))=1$ for every $\xi \in \Xi$. Then, we compute by (9) the Radon transform between the $\operatorname{SIM}(3)$-transitive spaces $X$ and $\Xi$ obtaining

$$
\mathcal{R} f(\theta, \varphi, \mathbf{t})=\int_{\mathbb{R}} f(t \mathbf{n}(\theta, \varphi)+\mathbf{t}) \mathrm{d} t,
$$

which is the integral of $f$ over the line parallel to the vector $\mathbf{n}(\theta, \varphi)$ and passing through the point $\mathbf{t} \in \mathbb{R}^{3}$. Let us now determine a suitable $\pi$-invariant subspace $\mathcal{A}$ of $L^{2}\left(\mathbb{R}^{3}\right)$ as in (A7). In order to do that, it is useful to derive a Fourier slice theorem for $\mathcal{R}$.

For any $f \in \mathcal{S}\left(\mathbb{R}^{3}\right)$, by Theorem 1.1 in [18, Chapter 2], we have

$$
(I \otimes \mathcal{F}) \mathcal{R} f(\theta, \varphi, \mathbf{v})=\mathcal{F} f(\mathbf{v}), \quad \mathbf{v} \in(\theta, \varphi)^{\perp} .
$$

As a consequence, by Plancherel theorem and formula (2.8) in [18, Chapter 7], we obtain

$$
\begin{aligned}
\|\mathcal{R} f\|_{L^{2}(\Xi)}^{2} & =\int_{0}^{\pi} \int_{0}^{\pi} \int_{(\theta, \varphi)^{\perp}}|(I \otimes \mathcal{F}) \mathcal{R} f(\theta, \varphi, \mathbf{v})|^{2} \sin \varphi \mathrm{d} \mathbf{v} \mathrm{d} \theta \mathrm{d} \varphi \\
& =\int_{0}^{\pi} \int_{0}^{\pi} \int_{(\theta, \varphi)^{\perp}}|\mathcal{F} f(\mathbf{v})|^{2} \sin \varphi \mathrm{d} \mathbf{v} \mathrm{d} \theta \mathrm{d} \varphi \\
& =\int_{\mathbb{R}^{3}} \frac{|\mathcal{F} f(\mathbf{v})|^{2}}{|\mathbf{v}|} \mathrm{d} \mathbf{v} .
\end{aligned}
$$

By using spherical coordinates, we obtain 


$$
\begin{aligned}
\|\mathcal{R} f\|_{L^{2}(\Xi)}^{2} & =\int_{0}^{\pi} \int_{0}^{\pi} \int_{\mathbb{R}}|\mathcal{F} f(\tau n(\theta, \varphi))|^{2}|\tau| \sin \varphi \mathrm{d} \tau \mathrm{d} \theta \mathrm{d} \varphi \\
& \leq \int_{0}^{\pi} \int_{0}^{\pi} \int_{|\tau| \leq 1}|\mathcal{F} f(\tau n(\theta, \varphi))|^{2} \sin \varphi \mathrm{d} \tau \mathrm{d} \theta \mathrm{d} \varphi \\
& +\int_{0}^{\pi} \int_{0}^{\pi} \int_{|\tau|>1}|\tau||\mathcal{F} f(\tau n(\theta, \varphi))|^{2} \sin \varphi \mathrm{d} \tau \mathrm{d} \theta \mathrm{d} \varphi \\
& \leq 4 \pi\|f\|_{1}^{2}+\|f\|_{2}^{2}<+\infty,
\end{aligned}
$$

which proves that $\mathcal{R} f \in L^{2}(\Xi)$ for any $f \in \mathcal{S}\left(\mathbb{R}^{3}\right)$ and we set $\mathcal{A}=\mathcal{S}\left(\mathbb{R}^{3}\right)$. Next, we show that $\mathcal{R}$, regarded as an operator from $\mathcal{A}$ to $L^{2}(\Xi)$ is closable. By [21, Theorem VIII.1], this is equivalent to proving that the adjoint of $\mathcal{R} f: \mathcal{A} \rightarrow L^{2}(\Xi)$ is densely defined. Suppose that $\left(f_{n}\right)_{n} \subseteq \mathcal{A}$ is a sequence such that $f_{n} \rightarrow f$ in $L^{2}\left(\mathbb{R}^{3}\right)$ and $\mathcal{R} f_{n} \rightarrow g$ in $L^{2}(\Xi)$. Since $I \otimes \mathcal{F}$ is unitary from $L^{2}(\Xi)$ onto $L^{2}(\Xi)$, we have that $(I \otimes \mathcal{F}) \mathcal{R} f_{n} \rightarrow(I \otimes \mathcal{F}) g$ in $L^{2}(\Xi)$. Since $f_{n} \in \mathcal{A}$, by $(37)$, for every $(\theta, \varphi) \in[0, \pi)_{*}^{2}$

$$
(I \otimes \mathcal{F}) \mathcal{R} f_{n}(\theta, \varphi, \mathbf{v})=\mathcal{F} f_{n}(\mathbf{v}), \quad \mathbf{v} \in(\theta, \varphi)^{\perp}
$$

Hence, passing to a subsequence if necessary,

$$
\mathcal{F} f_{n}(\mathbf{v}) \rightarrow(I \otimes \mathcal{F}) g(\theta, \varphi, \mathbf{v})
$$

for almost every $(\theta, \varphi) \in[0, \pi)_{*}^{2}$ and $\mathbf{v} \in(\theta, \varphi)^{\perp}$. Therefore, for almost every $(\theta, \varphi) \in$ $[0, \pi)_{*}^{2}$ and $\mathbf{v} \in(\theta, \varphi)^{\perp}$

$$
(I \otimes \mathcal{F}) g(\theta, \varphi, \mathbf{v})=\lim _{n \rightarrow+\infty} \mathcal{F} f_{n}(\mathbf{v})=\mathcal{F} f(\mathbf{v}),
$$

where the last equality holds true using a subsequence if necessary. Therefore, if $\left(h_{n}\right)_{n} \in \mathcal{A}$ is another sequence such that $h_{n} \rightarrow f$ in $L^{2}\left(\mathbb{R}^{3}\right)$ and $\mathcal{R} h_{n} \rightarrow h$ in $L^{2}(\Xi)$, then, for almost every $(\theta, \varphi) \in[0, \pi)_{*}^{2}$ and $\mathbf{v} \in(\theta, \varphi)^{\perp}$

$$
(I \otimes \mathcal{F}) h(\theta, \varphi, \mathbf{v})=\mathcal{F} f(\mathbf{v}) .
$$

Therefore,

$$
(I \otimes \mathcal{F}) g(\theta, \varphi, \mathbf{v})=(I \otimes \mathcal{F}) h(\theta, \varphi, \mathbf{v})
$$

for almost every $(\theta, \varphi) \in[0, \pi)_{*}^{2}$ and $\mathbf{v} \in(\theta, \varphi)^{\perp}$. Then $\lim _{n \rightarrow+\infty} \mathcal{R} f_{n}=\lim _{n \rightarrow+\infty} \mathcal{R} h_{n}$, and $\mathcal{R}$ is closable. We denote its closure by $\overline{\mathcal{R}}$.

\subsubsection{The unitarization theorem}

By Theorem 1 the Radon transform $\mathcal{R}: \mathcal{A} \rightarrow L^{2}(\Xi, \mathrm{d} \xi)$ admits a unique closure $\overline{\mathcal{R}}$ which satisfies 


$$
\overline{\mathcal{R}} \pi(\mathbf{b}, R, a)=\chi(\mathbf{b}, R, a)^{-1} \hat{\pi}(\mathbf{b}, R, a) \overline{\mathcal{R}}, \quad(\mathbf{b}, R, a) \in G,
$$

where $\chi(\mathbf{b}, R, a)=a$ since $\alpha(\mathbf{b}, R, a)=a^{3}, \beta(\mathbf{b}, R, a)=a$ and $\gamma(\mathbf{b}, R, a)=a^{2}$. Furthermore, there exists a unique positive self-adjoint operator

$$
\mathcal{I}: \operatorname{dom}(\mathcal{I}) \supseteq \operatorname{Im} \overline{\mathcal{R}} \rightarrow L^{2}(\Xi, \mathrm{d} \xi),
$$

semi-invariant with weight $\chi(\mathbf{b}, R, a)^{-1}=a^{-1}$ with the property that the composite operator $\mathcal{I} \overline{\mathcal{R}}$ extends to a unitary operator $Q: L^{2}(X, \mathrm{~d} x) \rightarrow L^{2}(\Xi, \mathrm{d} \xi)$ intertwining $\pi$ and $\hat{\pi}$, namely

$$
\hat{\pi}(g) Q \pi(g)^{-1}=Q, \quad g \in G .
$$

We can provide an explicit formula for $\mathcal{I}$. Consider the subspace

$$
\mathcal{D}=\left\{f \in L^{2}(\Xi): \int_{[0, \pi)_{*}^{2} \times(\theta, \varphi)^{\perp}}|\tau||(I \otimes \mathcal{F}) f(\theta, \varphi, \tau)|^{2} \sin \varphi \mathrm{d} \theta \mathrm{d} \varphi \mathrm{d} \tau<+\infty\right\}
$$

and define the operator $\mathcal{J}: \mathcal{D} \rightarrow L^{2}(\Xi)$ by

$$
(I \otimes \mathcal{F}) \mathcal{J} f(\theta, \varphi, \tau)=|\tau|^{\frac{1}{2}}(I \otimes \mathcal{F}) f(\theta, \varphi, \tau),
$$

a Fourier multiplier with respect to the variable $t$. A direct calculation shows that $\mathcal{J}$ is a densely defined positive self-adjoint injective operator and is semi-invariant with weight $\zeta(g)=\chi(g)^{-1}=a^{-\frac{1}{2}}$. By [7, Theorem 1], there exists $c>0$ such that $I=c \mathcal{J}$ and we now show that $c=1$. Consider a non-zero function $f \in \mathcal{A}$. Then, by Plancherel theorem, equation (37) and formula (2.8) in [18, Chapter 7], we obtain

$$
\begin{aligned}
\|f\|^{2}=\|\mathcal{I} \mathcal{R} f\|_{L^{2}(\Xi)}^{2} & =c^{2}\|(I \otimes \mathcal{F}) \mathcal{J} \mathcal{R} f\|_{L^{2}(\Xi)}^{2} \\
& =c^{2} \int_{[0, \pi)_{*}^{2} \times(\theta, \varphi)^{\perp}}|(I \otimes \mathcal{F}) \mathcal{R} f(\theta, \varphi, \tau)|^{2}|\tau| \sin \varphi \mathrm{d} \theta \mathrm{d} \varphi \mathrm{d} \tau \\
& =c^{2} \int_{[0, \pi)_{*}^{2} \times(\theta, \varphi)^{\perp}}|\mathcal{F} f(\tau)|^{2}|\tau| \sin \varphi \mathrm{d} \theta \mathrm{d} \varphi \mathrm{d} \tau \\
& =c^{2}\|f\|^{2} .
\end{aligned}
$$

Thus, $c=1$ and this concludes the proof.

\subsubsection{The inversion formula}

By Theorem 2, for any $f \in \mathcal{S}\left(\mathbb{R}^{3}\right)$, taking into account equations (26) and (27) and that $\chi(\mathbf{b}, R, a)=a^{\frac{1}{2}}$, the reconstruction formula (14) reads 


$$
f=\int_{\operatorname{SIM}(3)} a^{-5}\langle\overline{\mathcal{R}} f, \hat{\pi}(\mathbf{b}, R, a) \Psi\rangle_{L^{2}(\Xi, \mathrm{d} \xi)} \psi\left(a^{-1} R^{-1}(\mathbf{x}-\mathbf{b})\right) \mathrm{d} \mathbf{b} \mathrm{d} R \mathrm{~d} a,
$$

where the integral is weakly convergent.

Acknowledgements G.S. Alberti, F. De Mari and E. De Vito are members of the Gruppo Nazionale per l'Analisi Matematica, la Probabilità e le loro Applicazioni (GNAMPA) of the Istituto Nazionale di Alta Matematica (INdAM) and together with F. Bartolucci are part of the Machine Learning Genoa Center (MaLGa).

\section{References}

1. G. S. Alberti, F. Bartolucci, F. De Mari, E. De Vito. Unitarization and Inversion Formulae for the Radon Transform between Dual Pairs. SIAM J. Math. Anal. 51 (2019), no. 2, 4356-4381.

2. J.P. Antoine and R. Murenzi. Two-dimensional directional wavelets and the scale-angle representation. Signal Processing 52 (1996), no. 3, 259-281.

3. F. Bartolucci, F. De Mari, E. De Vito, F. Odone. The Radon transform intertwines wavelets and shearlets. Applied and Computational Harmonic Analysis 47 (2019), no. 3, 822-847.

4. C. Berenstein and D. Walnut. Local inversion of the Radon transform in even dimensions using wavelets. 75 years of Radon transform, Vienna, 1992, Conf. Proc. Lecture Notes Math. Phys., IV, Int. Press, Cambridge, MA (1994), 45-69.

5. N. Bourbaki. Integration II . Springer-Verlag, Berlin (2004).

6. S. Chern. On integral geometry in Klein spaces. Ann. of Math. (2) 43 (1942), 178-189.

7. M. Duflo and C. C. Moore. On the regular representation of a nonunimodular locally compact group. J. Functional Analysis 21 (1976), no. 2, 209-243.

8. H. Führ. Abstract harmonic analysis of continuous wavelet transforms. Springer, Berlin (2005).

9. H. Führ. Generalized Calderón conditions and regular orbit spaces. Colloq. Math. (1) 120 (2010), 103-126.

10. I. M. Gel'fand. Integral geometry and its relation to the theory of representations. Russian Math. Surveys 15 (1960), no. 2, 143-151.

11. S. Helgason. The Radon transform on Euclidean spaces, compact two-point homogeneous spaces and Grassmann manifolds. Acta Math. 113 (1965), 153-180.

12. S. Helgason. Geometric analysis on symmetric spaces. Mathematical Surveys and Monographs, American Mathematical Society 39, Providence, RI, 2nd ed. (2008).

13. S. Helgason. The Radon transform. Progress in Mathematics 5, Birkhäuser Boston, Inc., Boston, MA, 2nd ed. (1999).

14. S. Helgason. Some personal remarks on the Radon transform. Geometric analysis and integral geometry. Contemp. Math. 598, Amer. Math. Soc., Providence, RI (2013), 3-19.

15. M. Holschneider. Inverse Radon transforms through inverse wavelet transforms. Inverse Problems 7 (1991), 853-861.

16. W. R. Madych. Tomography, approximate reconstruction, and continuous wavelet transforms. Appl. Comput. Harmon. Anal. 7 (1999), 54-100.

17. S. Mostert Paul. Sections in principal fibre spaces. Duke Mathematical Journal 23 (1956), 57-71.

18. F. Natterer. The mathematics of computerized tomography. SIAM (2001).

19. T. Olson and J. DeStefano. Wavelet localization of the radon transform. IEEE Transactions on Signal Processing 42 (1994), 2055-2067.

20. J. Radon. Über die Bestimmung von Funktionen durch ihre Integralwerte längs gewisser Manigfaltigkeiten. Ber. Ver. Sächs. Akad. Wiss. Leipzig, Math Nat. Kl. 69 (1917), 262-277. 
21. M. Reed and B. Simon. Methods of Modern Mathematical Physics. I: Functional Analysis. Academic Press, New York, 2nd ed. (1980).

22. B. Rubin. The Calderón reproducing formula, windowed X-ray transforms, and Radon transforms in $L^{p}$-spaces. J. Fourier Anal. Appl. 4 (1998), 175-197.

23. D. C. Solmon. The X-Ray Transform. J. Math. Anal. Appl. 56 (1976), no. 1, 61-83.

24. V. S. Varadarajan. Lie Groups, Lie Algebras and Their Representations. Springer-Verlag, New York, 2nd ed. (1984).

25. V. S. Varadarajan. Geometry of quantum theory. Springer-Verlag, New York, 2nd ed. (1985).

26. D. Walnut. Local inversion of the Radon transform in the plane using wavelets. Proc. SPIE 2034 (1993), 84-90.

27. C. E. Yarman and B. Yazici. Euclidean motion group representations and the singular value decomposition of the Radon transform. Integral Transforms Spec. Funct. 18 (2007), 59-76. 